\title{
Photoinactivation of Glutamate Receptors by Genetically Encoded Unnatural Amino Acids
}

\author{
Viktoria Klippenstein, Valentina Ghisi, Marcus Wietstruk, and Andrew J. R. Plested \\ Leibniz Institut für Molekulare Pharmakologie, Berlin 13125, Germany, and Cluster of Excellence NeuroCure, Charité, Berlin 10117, Germany
}

Ionotropic glutamate receptors (iGluRs) are ubiquitous in the mammalian brain, and the AMPA-subtype is essential for fast, glutamateactivated postsynaptic currents. We incorporated photoactive crosslinkers into AMPA receptors using genetically encoded unnatural amino acid mutagenesis in a mammalian cell line. Receptors rescued by incorporation of unnatural amino acids, including $p$-benzoyl- $\mathrm{L}$ phenylalanine (BzF, also known as Bpa), had largely similar properties to wild-type channels and were expressed at similar levels. BzF incorporation at subunit interfaces afforded photocrosslinking of subunits, as assessed by biochemical experiments. In electrophysiological recordings, BzF incorporation allowed selective and potent UV-driven photoinactivation of both homomeric (GluA2) and heteromeric (GluA2:GluA1) AMPA receptors. State dependence of trapping at two sites in the lower lobe of the ligand binding domain is consistent with deformation of these domains as well as intersubunit rearrangements during AMPA receptor desensitization.

Key words: benzophenone; crosslinking; mammalian cells; orthogonal tRNA; synthetase; UV light

\section{Introduction}

Glutamate receptors (GluRs) are ubiquitous in the mammalian brain, and the AMPA subtype is essential for fast, glutamateactivated, postsynaptic currents (Traynelis et al., 2010). The four subunits in the receptor are drawn from four similar genes to form heterotetrameric complexes. Homotetramers also form, although these species are disfavored in vivo. Each subunit contributes to the membrane-spanning ion pore domain, and each has a ligand binding domain (LBD) and a distal N-terminal domain (ATD), which controls receptor assembly (Sobolevsky et al., 2009). Previous studies provided insight into the dynamics of subunit interfaces during receptor gating, using conventional cysteine mutagenesis (Armstrong et al., 2006; Weston et al., 2006b; Gielen et al., 2008; Plested and Mayer, 2009; Das et al., 2010; Lau et al., 2013). However, this method has limitations, including the requirement for two correctly placed cysteines. Disulfides may be well accommodated by isolated domains for crystallography; but when native cysteines are removed from the full-length receptor to improve specificity, expression suffers (Lau et al., 2013). Although absolute expression levels are of little importance for electrophysiological recordings, non-native ex-

Received Aug. 30, 2013; revised Nov. 13, 2013; accepted Dec. 3, 2013.

Author contributions:V.K. and A.J.R.P. designed research; V.K., V.G., M.W., and A.J.R.P. performed research;V.K. and A.J.R.P. analyzed data; V.K. and A.J.R.P. wrote the paper.

This work was supported by the Deutsche Forschungsgemeinschaft Grant EXC 257 (NeuroCure). V.K. was the recipient of a stipend from the Medical Neurosciences PhD program of the Charité Universitätsmedizin. We thank Thomas Sakmar for the gift of the tRNA and synthetase pairs; Drs. Stephan Sigrist, Michael C. Ashby, Chris Ahern, Sam Goodchild, and Stephan Pless for advice and helpful comments; Thomas Jentsch for the loan of a JML-C2 lamp; and Bjoern Schroeder for the loan of a UVICO lamp.

The authors declare no competing financial interests.

Correspondence should be addressed to Dr. Andrew J. R. Plested, Leibniz Institut für Molekulare Pharmakologie, Robert-Rössle-Strasse 10, Berlin 13125, Germany. E-mail: plested@fmp-berlin.de.

DOI:10.1523/JNEUROSCI.3725-13.2014

Copyright $\odot 2014$ the authors $\quad 0270-6474 / 14 / 340980-12 \$ 15.00 / 0$ pression and non-native disulfides are certain to bias the assembly of heteromeric complexes. In contrast, crosslinkers that react with primary amines can be used without mutagenesis (Cornell, 1989), but lack specificity both within and between targets.

With these limitations in mind, optically controllable crosslinkers introduced by unnatural amino acid (UAA) mutagenesis are attractive alternatives. The most common strategies for incorporating UAAs use nonsense suppression of Amber stop codons. Injection methods have been widely used for structure-function studies (Beene et al., 2003; Pless and Ahern, 2013), but this method requires synthesis and deprotection of tRNAs aminoacylated with the desired UAA, and conjugated tRNAs are depleted during incubation. Alternatively, an engineered orthogonal ami-

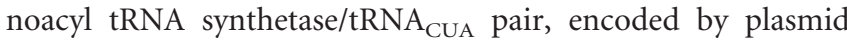
DNA, can be introduced to cells to suppress the stop codon (Wang et al., 2001; Chin et al., 2003). Numerous synthetases have been artificially evolved from prokaryotes to incorporate a range of UAAs (Liu et al., 2007b), in general with good orthogonality to their mammalian counterparts. UAAs carrying photoactive side chains have been studied both in vivo and in vitro (Chin et al., 2002a; Farrell et al., 2005; Hino et al., 2006) and have been deployed in neurons (Wang et al., 2007). Benzophenone has also been used extensively in peptides (Dormán and Prestwich, 1994) and conjugated to maleimide (Horn et al., 2000; Ding and Horn, 2001), and so its crosslinking reaction is well understood. However, there are few reports of photocrosslinking of membrane proteins or in mammalian cells (Ye et al., 2009, 2010, 2013; Naganathan et al., 2013).

In this study, we introduced $p$-benzoyl-L-phenylalanine (BzF) to the AMPA receptor subunit GluA2 using a genetically encoded orthogonal synthetase (Ye et al., 2008). This approach afforded highly efficient rescue of AMPA receptor expression and photo- 
inactivation of both homotetrameric and heterotetrameric receptors expressed in mammalian cells.

\section{Materials and Methods}

Molecular biology. We used the pRK5 expression vector encoding the flip splice variant of the rat GluA2 subunit with a QG background for studying homomeric receptors. In this vector, eGFP was expressed from an IRES site immediately after the GluA2 coding sequence. The endogenous TAG stop codon of GluA2 was edited to the Opal stop codon (TGA), and the Y40TAG mutation was introduced into eGFP to act as a reporter of cells that were competent for rescue of Amber codons (TAG; see Fig. 1A) (Liu et al., 2007b). Amber codons (at positions M721, G725, S729, and Y768 in GluA2) were introduced by overlap PCR, and the sequence of the entire amplified region was confirmed with double-stranded sequencing. GluA2 subunits with three cysteines deleted $(3 \times$ Cys $(-)$; C190A, C436S, C528S) and carrying a C-terminal FLAG-tag (Lau et al., 2013) were used as the background for making Amber mutants for biochemical experiments. For recordings of heteromeric receptors, we used rat GluA1 (Q) (without a fluorescent marker) in the pcDNA 3.1 $(+$ ) backbone, and the edited form of GluA2, A2 Q607R_IRES_eGFP-Y40TAG, with introduced Amber mutations at either G725 or S729.

Cell culture, transfection, and UAA incubation. GluRs were expressed in HEK-293 cells for electrophysiological recordings or in HEK-293T cells for Western blotting. Cells were maintained in MEM containing 10\% serum at $37^{\circ} \mathrm{C}$ and $5 \% \mathrm{CO}_{2}$. Transfections were performed using polyethylenimine in a $1: 3$ ratio (v/v; DNA/polyethylenimine). For experiments on homomeric receptors (including wild-type [WT] controls), we cotransfected GluA2 with vectors encoding mutated tRNA and aminoacyl-tRNA-synthetase pairs (gifts from Thomas Sakmar (Rockefeller University); Ye et al., 2008, 2009) in the mass ratio 4:1:1. These synthetases drive suppression of Amber codons by aminoacylating their cognate exogenous mutant tRNAs with either BzF or p-Azido-Lphenylalanine $(\mathrm{AzF})$. For recordings of heteromeric receptors, we cotransfected four vectors: (1) the rat GluA1 (Q) subunit; (2) the edited form of GluA2, A2 Q607R_IRES_eGFP-Y40TAG, carrying either the S729 or G725 amber mutation; (3) the BzF tRNA; and (4) the BzF tRNA synthetase. The DNA mass ratio (GluA1:GluA2) was typically 1:1. After $6 \mathrm{~h}$ of incubation, the transfection medium was replaced by MEM supplanted with an UAA. We dissolved BzF (Bachem Holding) in $1 \mathrm{M} \mathrm{HCl}$ and immediately added the solution to prewarmed MEM medium. AzF (Chem-Impex International) was dissolved in $1 \mathrm{M} \mathrm{NaOH}$. Media supplemented with $1 \mathrm{~mm} \mathrm{BzF}$ or $0.5 \mathrm{~mm} \mathrm{AzF}$ were adjusted to $\mathrm{pH} 7.3$ and filter-sterilized (0.22 $\mu \mathrm{m}$ PVDF filter) before use (based on Hino et al., 2006 and Ye et al., 2009).

Electrophysiology and photoinactivation. Outside-out patch recordings were performed $24-72 \mathrm{~h}$ after transfection. The external solution contained the following (in mM): $150 \mathrm{NaCl}, 0.1 \mathrm{MgCl}_{2}, 0.1 \mathrm{CaCl}_{2}, 5 \mathrm{HEPES}$. The pipette solution contained the following (in mM): $115 \mathrm{NaCl}, 10 \mathrm{NaF}$, $5 \mathrm{Na}_{4}$ BAPTA, $0.5 \mathrm{CaCl}_{2}, 1 \mathrm{MgCl}_{2}, 5$ HEPES, $10 \mathrm{Na}_{2}$ ATP. Both solutions were titrated to $\mathrm{pH} 7.3$ with $\mathrm{NaOH}$. The polyamine internal solution included the following (in mM): $120 \mathrm{NaCl}, 10 \mathrm{NaF}, 5 \mathrm{Na}_{4}$ BAPTA, 0.5 $\mathrm{CaCl}_{2}, 0.1$ spermine, 5 HEPES, and was titrated to $\mathrm{pH}$ 7.2. Glutamate (Sigma-Aldrich) and the allosteric modulator cyclothiazide (CTZ, Ascent Scientific) were diluted in the external solution. Fire-polished patch pipettes were mounted in a low noise holder (ISO-S-1.5G; G23 Instruments). We applied glutamate $(10 \mathrm{~mm})$ and, where appropriate, CTZ $(100 \mu \mathrm{M})$ to outside-out patches using a custom-made four-barrel glass perfusion tool (Vitrocom). The perfusion tool was mounted on a piezoelectric transducer (Physik Instrument), which was controlled via the digitizer interface (Instrutech ITC-18, HEKA Instruments). Except where noted, patches were clamped at $-60 \mathrm{mV}$. Currents (filtered at 5-10 kHz) were recorded using an Axopatch 200B amplifier and the Axograph program (Axograph Scientific). After establishment of fast perfusion recording conditions, the objective was switched to a $20 \times$ LUCPLFLN $20 \times \mathrm{Ph}$ lens (Olympus). The UV source was a $120 \mathrm{~W}$ metal halide lamp (UVICO; Rapp Optoelectronic). An integral shutter under computer control formed UV pulses of between 50 and 300 ms duration, which we passed through a short-pass $400 \mathrm{~nm}$ filter. A liquid light guide coupled the excitation light to a FC70 filter cube (in which a $420 \mathrm{~nm}$ dichroic mirror combined a $\sim 455 \mathrm{~nm}$ LED for visualizing GFP fluorescence) mounted on the epifluorescence port of an Olympus IX-71 microscope (see Fig. $3 A$, schematic). The UV power at the focal plane was $\sim 30 \mathrm{~mW}$ during full illumination (Gert Rapp, personal communication), or $\sim 30 \mathrm{~kW} / \mathrm{m}^{2}$. Several other UV sources we tried were either too dim to promote strong trapping on the timescale of our experiments (e.g., UV LED, $365 \mathrm{~nm}$, Thorlabs) or produced pulses that were too short to promote efficacious trapping (JML-C2 and SP-20, Rapp Optoelectronic). These observations suggest that abstraction of the hydrogen from nearby $\mathrm{C}-\mathrm{H}$ bonds by BzF is inefficient for the sites we have tried, in contrast to the very fast abstraction by Bpa-MTS in the S4 segment of the Shaker potassium channel (Horn et al., 2000) but in common with similar sites in the sodium channel (Ding and Horn, 2001). All control experiments on WT channels were done on the background of the cotransfected orthogonal BzF tRNA and tRNA synthetase, with cells incubated in the presence of BzF.

Biochemistry. For biochemical experiments, we used a GluA2 variant with three cysteines deleted that has a reduced tendency to dimerize in basal conditions (Lau et al., 2013). At $72 \mathrm{~h}$ after transfection, $10 \mathrm{~cm}$ dishes of cells were exposed to UVA light on ice in a ventilated chamber (Luzchem, LZC-1) for fixed time intervals. Intensities were 330-420 $\mathrm{W} / \mathrm{m}^{2}$, over a wider spectral band than in electrophysiological experiments because no short-pass filter was integrated into the chamber. To further reduce dimer formation from adventitious disulfide formation, we included 20-40 mM N-ethyl-maleimide (NEM, Thermo Fisher Scientific) during crosslinking. Cells were harvested immediately after UVA exposure and lysed in a buffer containing 1\% dodecylmaltoside (Glycon Biochemicals) and 20-40 mm N-ethyl-maleimide. The lysates were applied to M2-FLAG affinity beads (ANTI-FLAG M2 Affinity Gel, SigmaAldrich) and column purified (illustra MicroSpin, GE Healthcare). The second elution of material from the column was usually the most enriched in GluA2 protein. Eluted protein was loaded on $4-12 \%$ NuPAGE Novex gels (Invitrogen) and run in moderate reducing conditions (500 mM $\beta$-mercaptoethanol) at $180 \mathrm{~V}$ for $1 \mathrm{~h}$. Protein transfer to PVDF membranes was done in the XCell II Blot Module (Invitrogen) as described in the manufacturer's instructions. The membranes were incubated overnight at $4^{\circ} \mathrm{C}$ with the monoclonal mouse anti-FLAG M2 antibody (1:1000; Agilent Technologies). Membranes were incubated with the secondary antibody (goat anti-mouse IgG HRP, 1:5000; Cayman Chemical) for $1 \mathrm{~h}$ at room temperature. WT controls were expressed on the background of cotransfected BzF tRNA and tRNA synthetase and were incubated in an UAA-containing medium. Densitometric analyses were done with ImageJ. All chemicals were from Roth unless otherwise noted.

Data analysis. Deactivation and desensitization responses were fit with single exponential functions. Recovery from desensitization was fit with a Hodgkin-Huxley type function: $N=N_{0}+\left(1-N_{0}\right) *(1-$ $\exp (-k \mathrm{t}))^{n}$. The slope $(n)$ was usually 2 (Plested and Mayer, 2009). The rectification ratio (RR) was calculated as the ratio of the current at $-60 \mathrm{mV}$ to the current at $40 \mathrm{mV}$. IV curves were normalized to the peak current at $-60 \mathrm{mV}$ before UV application. Kymograms of peak current reduction were globally fit with exponential decay functions with a common steady-state level (where appropriate). Any discrepancy in terminal peak currents due to the potentiation of responses by CTZ was ignored. Statistical significance was assessed with Student's $t$ test, using either pairwise comparisons for different values from a single patch recording or unpaired tests for comparisons between mutants or different cells. For multiple comparisons, such as to assess the statistical significance of any differences between the kinetic properties of the Amber mutants tested and GluA2 S729Y (eight groups in all, including WT GluA2 as the control group), or the time constants of current reduction in crosslinking experiments, we performed oneway ANOVA with Dunnett's post hoc test, where appropriate. In biochemical analyses, the dimer fraction was calculated as the fraction of the dimer band intensity divided by the sum of the dimer and monomer band intensities. 


\section{Results}

Amber mutant receptors are rescued by photoactivatable amino acids

For initial characterization of genetic suppression of Amber (TAG) stop codons in glutamate receptors with photoactivatable UAAs, we chose sites within GluA2 in the lower (D2) lobes of the LBD (S729, G725; Fig. 1). These interfacial sites have been previously shown to form intermolecular disulfide crosslinks that inhibit the receptor (Armstrong et al., 2006; Plested and Mayer, 2009). Cells were transfected with the mixture of tRNA, tRNA synthetase, and GluA2_IRES_eGFP-Y40TAG plasmids (with GluA2 harboring the S729TAG or G725TAG mutation) and were incubated in the presence of $0.5-1$ $\mathrm{mM}$ BzF or p-azido-L-phenylalanine $(\mathrm{AzF})$. In these conditions, cells that showed green fluorescence contained rescued eGFP (and therefore also a functional aminoacyl-tRNA synthetase/ tRNA $_{\text {CUA }}$ pair). Such cells invariably gave large responses to $10 \mathrm{~mm}$ glutamate, with peak currents in outside-out patches of $-470 \pm 50 \mathrm{pA}$ (at $-60 \mathrm{mV}$; $n=50)$ for GluA2 S729BzF and $-620 \pm$ 90 pA for GluA2 G725BzF $(n=43)$. We presume that the vast majority of this current arises from rescued receptors harboring the non-natural photoactive amino acid, because subunits truncated within the S2 segment are unlikely to form functional receptors. These observations (and our biochemical results, see below) suggest that the synthetases we have used incorporate $\mathrm{BzF}$ and $\mathrm{AzF}$ with high efficiency and have a high degree of orthogonality to mammalian synthetases (Ye et al., 2008; 2010).

\section{Functional properties of AMPA receptors incorporating AzF and $\mathrm{BzF}$}

Patch-clamp electrophysiology coupled to rapid perfusion demonstrated that BzF-rescued GluRs had kinetic properties akin to WT GluRs. Rates of desensitization for the GluA2 mutants S729BzF $\left(k_{\text {des }}\right.$ $\left.=186 \pm 9 \mathrm{~s}^{-1}, n=20\right)$ and $\operatorname{G7} 25 \mathrm{BzF}\left(k_{\mathrm{des}}=197 \pm 8 \mathrm{~s}^{-1}, n=22\right)$ were modestly increased $(\sim 1.5$-fold) compared with WT GluA2 expressed on the background of BzF and the tRNA/tRNA-synthetase pair $\left(k_{\mathrm{des}}=121 \pm 6 \mathrm{~s}^{-1}, n=22\right.$; Fig. $\left.2 A, B\right)$. Small increases in the desensitization rate are also seen for cysteine mutants at these positions (Plested and Mayer, 2009). Surprisingly, rescuing the Amber stop codon with AzF at position S729 produced receptors with approximately eightfold faster desensitization kinetics $\left(k_{\text {des }}=930 \pm 80\right.$ $\left.\mathrm{s}^{-1}, n=15\right)$ compared with WT GluA2 $(p<0.0001)$. This effect was not observed with incorporation of AzF at position $\mathrm{G} 725\left(k_{\mathrm{des}}=\right.$ $\left.171 \pm 15 \mathrm{~s}^{-1}, n=9\right)$. The similar natural substitution of tyrosine (S729Y) produced a receptor with fourfold faster desensitization $\left(k_{\mathrm{des}}=420 \pm 40 \mathrm{~s}^{-1}, n=5 ; p<0.0001\right)$ than WT receptors. The deactivation rates for $\mathrm{BzF}$ mutants $\left(\mathrm{S} 729 \mathrm{BzF} k_{\text {deact }}=1500 \pm 120\right.$ $\mathrm{s}^{-1}, n=22$; G725BzF $\left.k_{\text {deact }}=1400 \pm 200 \mathrm{~s}^{-1}, n=15\right)$ were indistinguishable from those of WT receptors $\left(\mathrm{WT} k_{\text {deact }}=1600 \pm 150\right.$ $\mathrm{s}^{-1}, n=13$; Fig. 2C,D). The rate of recovery from desensitization for $\operatorname{S729BzF}\left(k_{\text {rec }}=36 \pm 3 \mathrm{~s}^{-1}, n=14\right)$ was also indistinguishable from
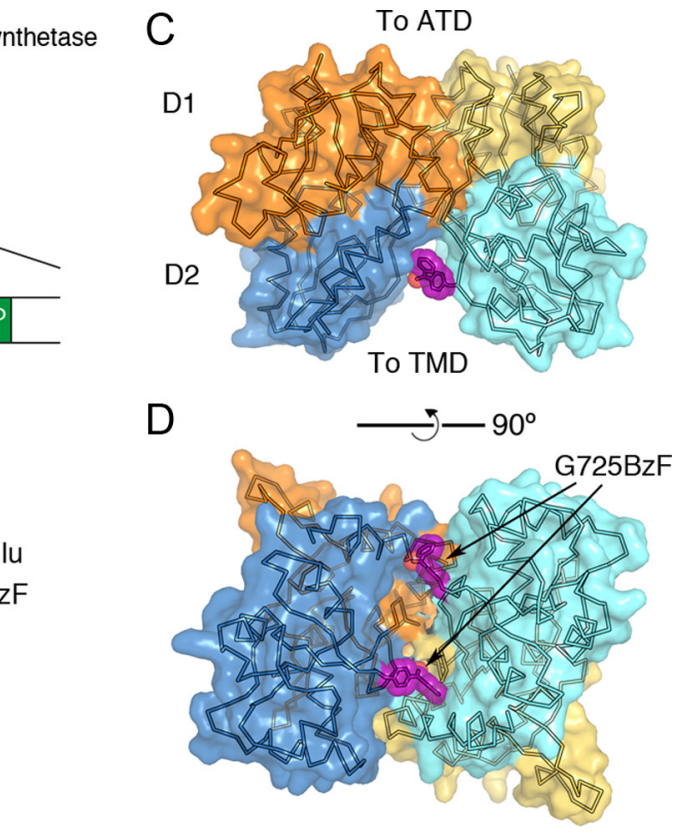

tRNA-Synthetase
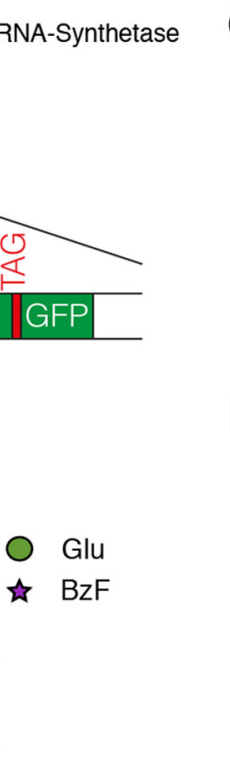

Figure 1. Sites of UAA incorporation and the design of the bicistronic dual Amber reporter. $\boldsymbol{A}, \mathrm{A}$ single mRNA encodes GluA2 and al., 2007a) and was inserted at position 725 instead of glycine. D, View of the same dimer as in C from below. Both BzF side chains at position 725 are shown within the dimer.

that of WT receptors $\left(k_{\mathrm{rec}}=40 \pm 3 \mathrm{~s}^{-1}, n=10\right.$; Fig. $\left.2 E, F\right)$ but detectably slowed for $\mathrm{G} 725 \mathrm{BzF}\left(k_{\mathrm{rec}}=23 \pm 2.5 \mathrm{~s}^{-1}, n=8 ; p=0.03\right)$. These limited differences suggest that the substitutions had little effect on glutamate affinity (Robert et al., 2005; Weston et al., 2006a).

It is possible that the distinct kinetics of the $\mathrm{AzF}$ and $\mathrm{BzF}$ mutants at S729 arise from different random mixtures of endogenous and UAAs that are incorporated at these sites by the specific synthetases. However, our subsequent photocrosslinking experiments, highly efficient rescue as assessed by biochemistry, and the weak effects of other natural amino acids introduced at this site, strongly argue that the genetic method of incorporation is specific. Thus, we presume that the intended UAAs were incorporated, rather than a random selection. The strong divergence of S729AzF kinetics from those of WT receptors, the known limitations of Azido groups as photocrosslinkers (see Discussion), and the potential of BzF substitutions to be functionally silent in native receptors led us to concentrate exclusively on BzF rescue for subsequent experiments.

\section{Irreversible inactivation of GluA2 BzF mutants by UV light}

We exposed outside-out patches from cells expressing GluA2 (carrying BzF at position G725 or S729) to pulses of UV light during applications of $10 \mathrm{~mm}$ glutamate (Fig. $3 A$ ). The pulse of UV was started $\sim 100 \mathrm{~ms}$ after the peak of the current, by which point $>90 \%$ of receptors were in the desensitized state. After $50-200 \mathrm{~ms}$ exposures with a repetition frequency of $0.5 \mathrm{~Hz}$, we detected a remarkably consistent peak current reduction for both Amber mutants tested: GluA2 S729BzF (Fig. 3 B, C) and GluA2 G725BzF (Fig. 3D,E). The rate of current reduction depended on UV intensity and exposure time, with longer applications and 


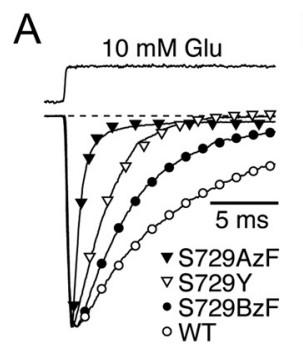

E

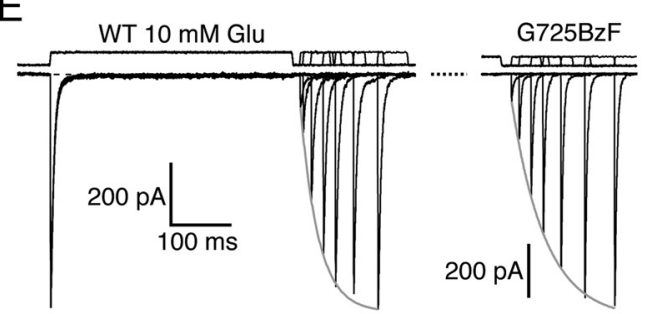

C

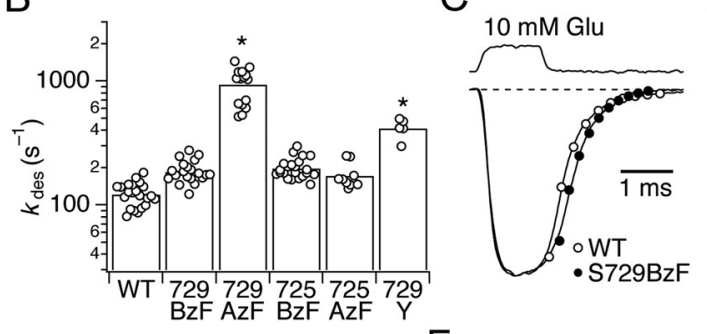

$F$

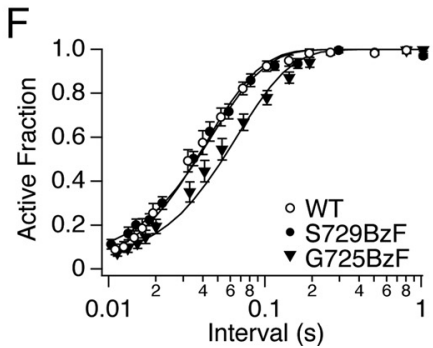

Figure 2. Kinetic properties of GluA2 receptors harboring UAAs. $A$, The rate of desensitization for the GluA2 S729BzF mutant (here, $k_{\text {des }}=252 \mathrm{~s}^{-1}$ ) was similar to WT GluA2 $\left(k_{\text {des }}=135 \mathrm{~s}^{-1}\right.$ in this example). Incorporation of AzF at position 5729 resulted in 10 -fold faster desensitization kinetics (for this trace, $k_{\text {des }}=1470 \mathrm{~s}^{-1}$ ). The $S 729 \mathrm{Y}$ mutant had fourfold faster desensitization ( $k_{\text {des }}=$ $435 \mathrm{~s}^{-1}$ ) than WT. $B$, Summary of desensitization rates of receptors carrying BzF or AzF (WT $k_{\text {des }}=121 \pm 6 \mathrm{~s}^{-1}, n=22 ; \mathrm{S729BzF} k_{\text {des }}=186 \pm 9 \mathrm{~s}^{-1}, n=20 ; \mathrm{G} 725 \mathrm{BzF} k_{\text {des }}=197 \pm 8 \mathrm{~s}^{-1}$, $n=22$ ). One-way ANOVA gave $p=0.25$ for $S 729 \mathrm{BzF}$ and $p=0.11$ for $\mathrm{G} 725 \mathrm{BzF}$ (WT was the control group). AzF at S729 produced receptors with $k_{\text {des }}$ of $930 \pm 80 \mathrm{~s}^{-1}$ ( $\left.n=15:{ }^{*} p<0.0001\right)$. AzF at position $\mathrm{G} 725$ gave $k_{\text {des }}$ of $171 \pm 15 \mathrm{~s}^{-1}(n=9 ; p=0.75)$. S729Y produced receptors with $k_{\text {des }}$ of $420 \pm 40 \mathrm{~s}^{-1}\left(n=5:{ }^{*} p<0.0001\right)$. C, Deactivation after a brief $(\sim 1 \mathrm{~ms})$ pulse of $10 \mathrm{~mm}$ glutamate for GluA2 S729BzF and G725BzF mutants was similar to WT GluA2 (here, $k_{\text {deact }}=1910 \mathrm{~s}^{-1}$ for WT and $k_{\text {deact }}=1730 \mathrm{~s}^{-1}$ for S729BzF). D, Summary of deactivation rates with BzF inserted at positions 5729 or G725 (S729BzF $\left.k_{\text {deact }}=1500 \pm 120 \mathrm{~s}^{-1}, n=22 ; \mathrm{G} 725 \mathrm{BzF} k_{\text {deact }}=1400 \pm 200 \mathrm{~s}^{-1}, n=15\right)$, which were indistinguishable from those of WT receptors (WT $k_{\text {deact }}=1600 \pm$ $150 \mathrm{~s}^{-1}, n=13 ; p=0.98$ for $\mathrm{S729BzF}$ and $p=0.86$ for $\left.\mathrm{G} 725 \mathrm{BzF}\right)$. $\boldsymbol{E}$, Seven overlaid traces show that recovery from desensitization for $\mathrm{G} 725 \mathrm{BzF}$ (here, $k_{\text {rec }}=46 \mathrm{~s}^{-1}$ ) was similar to WT GluA2 $\left(k_{\text {rec }}\right.$ $\left.=43 \mathrm{~s}^{-1}\right) . \boldsymbol{F}$, Summary of recovery rates of WT $\left(k_{\text {rec }}=40 \pm 3 \mathrm{~s}^{-1}, n=10\right)$ and the BzF mutants $\mathrm{G} 725\left(k_{\text {rec }}=23 \pm 2.5 \mathrm{~s}^{-1}, n=8:{ }^{*} p=0.03\right)$ and S729 $\left(k_{\text {rec }}=36 \pm 2.8 \mathrm{~s}^{-1}, n=14 ; p=0.89\right)$.

higher intensities resulting in faster trapping time courses, for both Amber mutants (Fig. $3 B, D, F$ ). Pausing the UV exposures stopped the peak current reduction, with subsequent responses in the absence of UV pulses having stable amplitudes over many episodes, excluding nonspecific rundown of the peak current as a cause of the inhibition. Photoinactivation of mutant receptors occurred with the time constant of $3.3 \pm 0.4 \mathrm{~s}$ cumulative UV exposure for GluA2 S729BzF if UV pulse trains of $50 \mathrm{~ms}$ were used $(n=19)$ and with a constant of $4.4 \pm 0.4 \mathrm{~s}$ for $200 \mathrm{~ms}$ pulses $(n=9$, probability of no difference $=0.07 ;$ Fig. $3 F)$. The trapping half-times for GluA2 G725BzF were $2.8 \pm 0.3 \mathrm{~s}$ for short pulses of $50 \mathrm{~ms}(n=9)$ and $4.4 \pm 0.8 \mathrm{~s}$ for $200 \mathrm{~ms}$ pulses $(n=9 ; p=0.08)$. In general, we were able to photoinactivate the receptors by $90-$ $95 \%$ with $\sim 10$ s of cumulative exposure to UV $(\sim 365 \mathrm{~nm})$. The initial amplitude of the GluA2 current correlated weakly, if at all, with the extent of receptor trapping upon cumulative UV exposures of 20 s for either S729BzF and G725BzF, with similar degrees of peak current reduction for a 100 -fold range of current amplitudes $\left(R^{2}{ }_{\text {S729BzF }}=0.3, n=10 ; R^{2}{ }_{\text {G725BzF }}=0.03, n=12\right.$; data not shown). The time course of reduction in peak current was invariably well fit with a single exponential, independent of the conditions or the intensity of the illumination (Fig. $3 B, D$ ), suggesting that a single rate-limiting step (e.g., the trapping of one LBD dimer in an inactive conformation by a single crosslink) produces the inhibition. More complicated scenarios involving multiple independent trapping events could also produce a single exponential (see Discussion). Notably, the exposure of UV did not affect the kinetics of homomeric receptors. For example, the rate of desensitization for the S729BzF mutant after $14 \mathrm{~s}$ of UV was $204 \pm 7 \mathrm{~s}^{-1}$, compared with $238 \pm 33 \mathrm{~s}^{-1}$ before UV in the same patch $(n=4)$. WT channels expressed on the background of tRNA, the tRNA synthetase, and BzF were comparatively unaffected by similar UV exposures (Fig. 3G,H). Extended UV exposures of at least $14 \mathrm{~s}$ were accompanied by peak current reduction of only $10 \pm$
$1 \%(n=10)$, which was not related to intensity or cumulative exposure and was more likely to be a result of peak current rundown during these long-lasting recordings.

To understand the basis of the untrappable current, we investigated whether endogenous amino acids are incorporated by the BzF synthetase or whether the inserted Amber stop codon was ineffective in terminating translation. Expression of TAG mutant GluA2 subunits alone (with the eGFP-TAG reporter) yielded no green cells, confirming that TAG stop codons do effectively block translation in HEK cells. However, consistent with previous reports (Wang et al., 2007), we observed $\sim 10-20$ faintly green cells per $8 \mathrm{~mm}$ coverslip, when mutant subunits were expressed on the background of the tRNA and tRNA synthetase, but without the UAA in the culture medium. Unsurprisingly, given that eGFP was rescued in these cells, after extended culture intervals (3-7 d), patches from these cells yielded glutamate-activated currents, presumably from GluA2 subunits rescued by nonspecific incorporation of endogenous amino acids. These currents were typically small ( $\leq 200 \mathrm{pA})$. Critically, and similarly to WT channels expressed on the BzF synthetase and tRNA background, these glutamate-activated currents were unaffected by long-lasting UV exposures $(n=4$ patches from G725 and S729 TAG mutants each). As our biochemical results confirm (see below and Discussion), these low levels of readthrough are not consistent with a detectable population of tetrameric receptors that completely lack BzF groups, suggesting that the residual current is principally a property of trapped receptors.

Overall, incorporating the UAA BzF in the lower lobes of the LBD of GluA2 endowed the receptors with specific and rapid UV-driven photoinactivation.

\section{Photoinactivation of heteromeric AMPA receptors}

We reasoned that, because a single trapping event per complex might be sufficient to abolish $\sim 95 \%$ of the current response of 
A
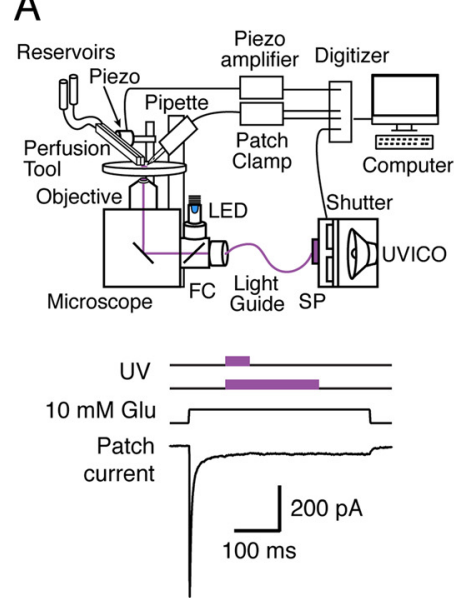

$\mathrm{F}$

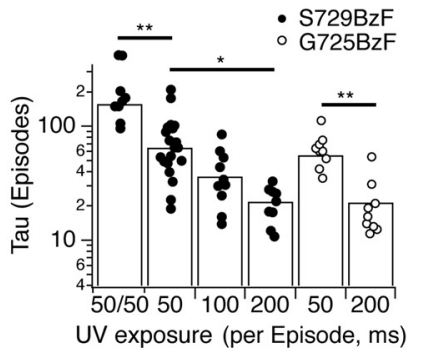

B

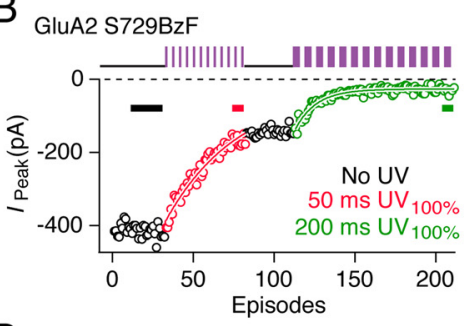

D GluA2 G725BzF

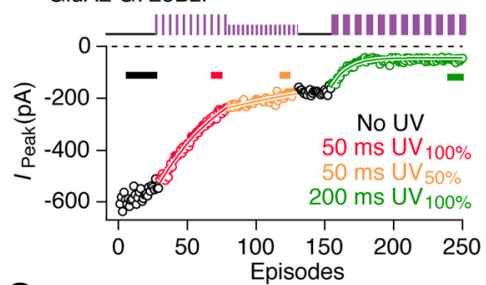

$G$

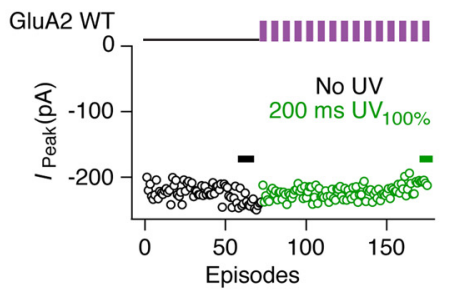

C

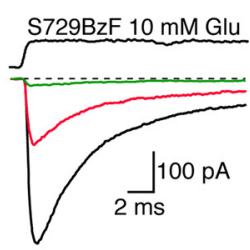

$\mathrm{E}$

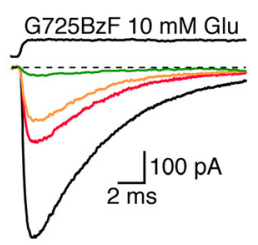

$\mathrm{H}$

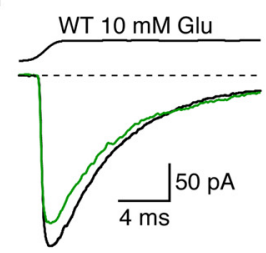

Figure 3. UV-induced reduction in peak current. $A$, Diagram of the setup used for electrophysiology coupled to photoinactivation. SP, SP400 short path filter; $\mathrm{FC}$, filter cube. The path of UV light is indicated as a violet beam. Traces indicate the responses of outside-out patches to $10 \mathrm{~mm}$ glutamate applications and the timing of their exposure to UV (examples of 50 or $200 \mathrm{~ms}$ pulses are shown) during each episode. The repetition frequency was $0.5 \mathrm{~Hz}$. The intensity of UV illumination was varied between either $50 \%$ or $100 \%$. B. Example of the time course of receptor photoinactivation in full UV illumination for GluA2 S729BzF. Periods during which the patch was serially exposed to UV exposures ( 50 or $200 \mathrm{~ms}$ ) are indicated schematically by violet pulse trains (not representative of the actual number of exposures). The circles represent the peak current activated by $10 \mathrm{~mm}$ glutamate in each episode. The rate of trapping was monoexponential (white outlined fits). The trapping rate was approximately threefold faster for the application of $200 \mathrm{~ms}$ pulses ( $\tau=13$ episodes; shown as green circles) compared with shorter pulses of $50 \mathrm{~ms}$ ( $\tau=45$; red). There is a lack of change in the current amplitude during brief periods without UV exposure (black circles). C, Example traces for GluA2 S729BzF illustrate the peak current reduction induced by UV exposures. The averages of $5-20$ responses to $10 \mathrm{~mm}$ glutamate from the stretches indicated with bars in $\boldsymbol{B}$ are overlaid. $\boldsymbol{D}$, For GluA2 G725BzF, the time course of trapping during a similar experiment to that in $\boldsymbol{B}$ is shown, with trapping rates similar to $5729 \mathrm{BzF}$ ( $\tau_{50 \mathrm{~ms}}=53$ episodes, red circles; $\tau_{200 \mathrm{~ms}}=14$ episodes, green). An additional series of exposures at $50 \%$ intensity was applied $\left(\tau_{50 \mathrm{~ms}}=144\right.$ episodes, orange). $\boldsymbol{E}$, Equivalent to $\boldsymbol{C}$, showing example average traces for GluA2 G725BzF during photoinactivation. Averaged stretches are indicated in $\boldsymbol{D}$ by color-coded bars. $\boldsymbol{F}$, Summary of the exponential half-times (in episodes) of G725BzF/S729BzF mutant inactivation, plotted against the UV exposure periods per episode in milliseconds. The intensity of UV illumination was $100 \%$, unless otherwise noted. The average time constants obtained for GluA2 S729BzF were as follows: $\tau_{50 \mathrm{~ms} 50 \%}=160 \pm 27, n=9 ; \tau_{50 \mathrm{~ms}}=65 \pm 8, n=19 ; \tau_{100 \mathrm{~ms}}=36 \pm 6, n=10 ; \tau_{200 \mathrm{~ms}}=22 \pm 2, n=9$. One-way ANOVA gave $p_{50 \mathrm{~ms} 50 \%}<0.001 ; p_{100 \mathrm{~ms}}=0.21 ; p_{200 \mathrm{~ms}}=0.04$ (50 ms exposure at $100 \%$ intensity was the control group). GluA2 G725 produced the following trapping rates: $\tau_{50 \mathrm{~ms}}=$ $56 \pm 6, n=9 ; \tau_{200 \mathrm{~ms}}=22 \pm 4, n=9, p=0.0002$ (Student's $t$ test). The BzF mutants did not differ in regard to their rates of trapping at given exposure times (probability of no difference for $50 \mathrm{~ms}$ exposures $=0.38$; for $200 \mathrm{~ms}$ pulses, $p=0.96$ ). ${ }^{*} p<0.05 .{ }^{*} p<0.001$. G, Kymogram of the peak current responses of WT receptors before and after exposure to UV (with $200 \mathrm{~ms}$ pulses per episode, green markers). WT receptors were unaffected by a cumulative UV exposure of $20 \mathrm{~s}$. $\boldsymbol{H}$, Example pre- and post-UV traces for WT receptors. Averages were done as in $\boldsymbol{C}$.

homomeric GluA2 receptors, the inclusion of at least one subunit harboring a photoactive crosslinker should effectively inactivate heteromeric complexes containing naive subunits. To investigate this possibility, we cotransfected the WT GluA1 (Q) subunit along with GluA2 (R) S729 or G725 Amber mutant subunits and tRNA and tRNA synthetase vectors. For the GluA2 (R) subunit, an IRES_eGFP-Y40TAG mutant vector was used, whereas the GluA1 vector did not encode any fluorescent reporter. We clamped patches at $40 \mathrm{mV}$ during fast perfusion electrophysiology to block homomeric GluA1 Q-containing channels with 30 $\mu \mathrm{M}$ spermine (see Materials and Methods). In these conditions, green cells that gave currents after incubation in BzF were likely to express A1:A2 heteromeric receptors with BzF substitutions only on the GluA2 subunit because homomeric receptors formed from GluA2 (R) subunits show weak functional expression (see below). We compared responses at positive voltages with those of the potentially mixed population at $-60 \mathrm{mV}$. Neither homomeric WT GluA1 controls $(n=4)$ nor heteromeric WT receptors containing A1 (Q) and A2_IRES_eGFP-Y40TAG (R) subunits $(n=4)$ exhibited UV-dependent reduction in current (Fig.
$4 A, B)$. However, consistent with crosslinking of homomeric receptors, UV exposure caused a monoexponential loss of the peak receptor current at positive potentials for heteromeric receptors with either BzF mutant, A1:A2 S729BzF (Fig. 4C,D) or A1:A2 G725BzF (Fig. 4E,F). The maximum extent of current reduction was less than that for homomeric mutant receptors. After $20 \mathrm{~s}$ of cumulative UV exposure, A1:A2 S729BzF receptor peak currents were reduced by $65 \pm 3 \%$ ( $p=0.007$ compared with homomeric GluA2 (Q) receptors; $n=11$ ), and for G725BzF the reduction was $73 \pm 4 \%(p=0.014$ vs homomeric GluA2 $(\mathrm{Q}) ; n=10)$. As for the homomeric counterparts, these extents were not significantly different from each other $(p=0.14$; Fig. $4 G)$. The time constants for current reduction of $4.7 \pm 0.4 \mathrm{~s}$ of cumulative UV exposure for A1:A2 S729BzF $(n=9)$ and $4.9 \pm 0.8 \mathrm{~s}$ for A1:A2 G725BzF ( $n=9$; Fig. $4 H$ ) did not significantly differ between the mutants $(p=0.84)$ and were similar to the time constants obtained for homomeric receptors under similar UV conditions. As for homomeric GluA2 receptors, the reduction of the peak current upon $20 \mathrm{~s}$ cumulative UV exposures was only weakly related to the initial current amplitude $\left(R_{\mathrm{S} 729 \mathrm{BzF}}^{2}=0.1, n=12 ; R_{\mathrm{G} 725 \mathrm{BzF}}^{2}=0.62\right.$, 
A

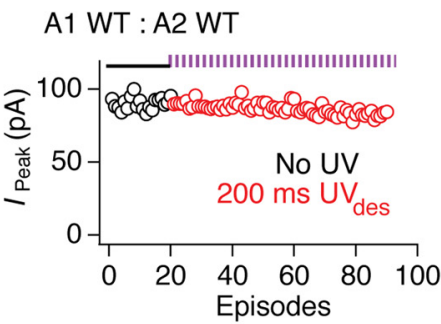

C

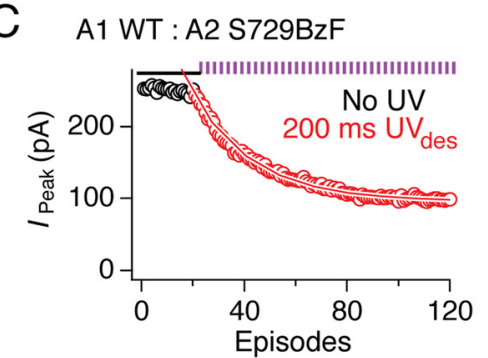

E

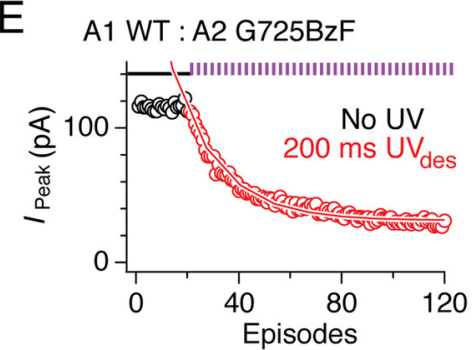

G

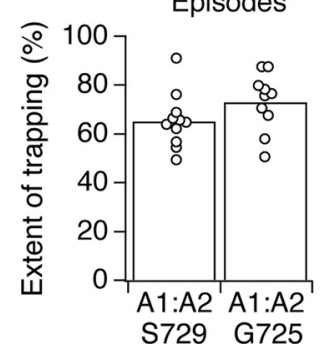

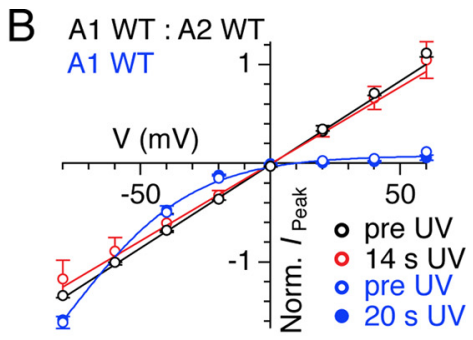
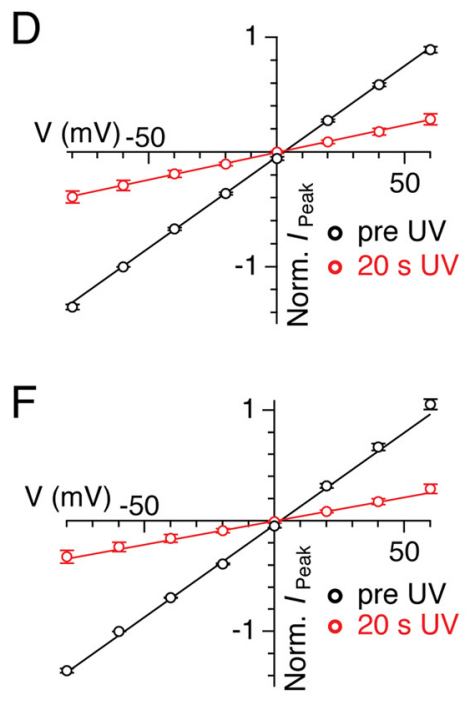

$\mathrm{H}$

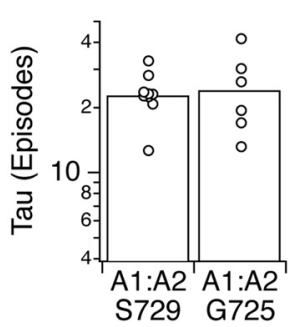

Figure 4. Heteromeric receptors can be photoinactivated. $\boldsymbol{A}$, Example kymogram for WT GluA1:GluA2 heteromeric receptors exposed to $200 \mathrm{~ms}$ pulses of UV in the desensitized state. Cumulative exposure of UV for this example was $14 \mathrm{~s}$ (indicated schematically as a pulse train; violet). Each marker (black, pre-UV; red, during UV application) represents the peak current during the appropriate episode. $\boldsymbol{B}$, IV relations for GluA1 WT (blue curves) and WT GluA2:GluA1 heteromeric receptors (black curves for pre-UV; red curve for post-UV) in the presence of $30 \mu \mathrm{m}$ spermine. The $20 \mathrm{~s}$ of cumulative UV did not induce any reduction in peak current for either condition ( $n=4$ for each IV relation). C, Example kymogram for the same experimental procedure as in $A$ on $A 2$ S729BzF:A1 receptors. The time course of trapping was monoexponential (white outlined fits), and the time constant for peak current reduction in this example was 24 episodes ( $4.8 \mathrm{~s} \mathrm{UV}$ ). The extent of trapping after a cumulative UV exposure of $20 \mathrm{~s}$ was $61 \%$. D, IV relation before and after UV-induced photoinactivation for A2 S729BzF:A1. The linear IV relation (black curve) before UV exposure indicates a predominately heteromeric population of receptors $\left(R R_{\text {pre uv }}=1.7 \pm 0.04 ; n=12\right)$. UV exposures of 20 s did not induce rectification (red curve; $R R_{\text {post uv }}=1.7 \pm 0.1 ; n=8 ; p=0.72$ vs before UV). $\boldsymbol{E}$, As in $\boldsymbol{A}$, an example kymogram for $\mathrm{A} 2$ G725BzF:A1 receptors exposed cumulatively to $20 \mathrm{~s} U V$. The time course of trapping was monoexponential (white outlined fits) and the time constant of the current reduction for this example was 18 episodes (3.6 $\mathrm{s} \mathrm{UV}$ ), resulting in inhibition of the peak current by $74 \%$. F, IV relation before and after UV-induced photoinactivation for A2 G725BzF:A1. The IV relation was linear before (black circles; $R R_{\text {pre Uv }}=1.5 \pm 0.1, n=13$ ) and after UV exposure ( $20 \mathrm{~s}$; red circles; $R R_{\text {post } \mathrm{Uv}}=1.4 \pm 0.2, n=7 ; p=0.5$ vs before UV). $\mathbf{G}$, Summary of the extent of receptor trapping upon 20 s of cumulative UV. $\boldsymbol{H}$, Summary of the exponential half-times (in episodes) for both BzF-containing heteromeric receptors. The average $\tau$ for S729BzF:A1 was $23 \pm 2$ episodes $(n=9)$ and for G725BzF:A1 receptors, $\tau=25 \pm 4$ episodes $(n=6)$. The photoinactivation time course was not significantly different between the mutants $(p=0.84)$

$n=10$; data not shown). We confirmed that the S729BzF mutation did not generate a large contaminant homomeric GluA2 (R) population. Homomeric GluA2 S729BzF (R) receptors gave very small responses $48-72 \mathrm{~h}$ after transfection (mean $-2.1 \pm 0.7 \mathrm{pA}$ at -60 $\mathrm{mV}, n=9$ ) in the presence of $30 \mu \mathrm{M}$ spermine (range, 0 to $-5.8 \mathrm{pA}$ ); four of these patches gave no detectable response, whereas the same mutant on the homomeric Q-background gave substantial

currents $(-240 \pm 73 \mathrm{pA}, n=8$; recorded on the same day). Any contamination with homomeric GluA1 channels was limited because, for most patches, the IV relation was almost linear, with RR (see Materials and Methods) of $1.7 \pm 0.04$ for A1:A2 S729BzF $(n=12$; Fig. $4 D)$ and $1.5 \pm 0.1$ for A1:A2 G725BzF $(n=13$; Fig. $4 F)$. The RRs did not evolve with continuing application $(\sim 20 \mathrm{~s})$ of UV for $\mathrm{A} 1: \mathrm{A} 2 \mathrm{G} 725 \mathrm{BzF}\left(R R_{\text {postUV }}=\right.$ $1.4 \pm 0.2, n=7, p=0.5$ vs before UV) or $\mathrm{A} 1: \mathrm{A} 2 \mathrm{~S} 729 \mathrm{BzF}\left(R R_{\text {postUV }}=1.7 \pm 0.1, n=\right.$ $8, p=0.72)$. In some patches, we obtained higher initial RRs, consistent with a largely homomeric $\mathrm{A} 1$ receptor population $(R R>$ $2)$. In this case, a substantial proportion of the receptors were homomeric GluA1, and selective inactivation of heteromers by UV light led to an increasingly rectifying IV relation, as expected (increase in $R R$ for $\mathrm{S} 729 \mathrm{BzF}$ was $30 \pm 6 \%$; range of initial RRs was $2.7-5.8, n=4)$. The current reduction at $40 \mathrm{mV}$, after $20 \mathrm{~s} \mathrm{UV}$ exposure, was independent of the RR (linear regression gave $R^{2}=0.04$ for A1:A2 S729BzF; range of $R R$ values 1.5-5.8), consistent with effective isolation of BzF-containing heteromers by spermine.

Together, these results demonstrate that BzF substitutions can photoinactivate heteromeric glutamate receptors in a subunit-specific manner, which could be useful in native cells.

\section{UV-induced crosslinking of subunits by $\mathrm{BzF}$ in live cells}

Despite the precedent of inhibition through disulfide crosslinking at D2 sites (Armstrong et al., 2006; Plested and Mayer, 2009), we sought to confirm that UV exposure controls receptor kinetics by physically linking subunits. Given that, after UV activation, benzophenone crosslinks to any C-H bond in good context (Dormán and Prestwich, 1994), it was conceivable that BzF made purely intrasubunit covalent bonds that render receptors inactive by distorting local protein structure. To examine the propensity of BzF to physically crosslink receptor subunits at sites in the LBDs, we performed biochemical experiments after in vivo photocrosslinking.

To ensure a monomeric background in reducing SDS-PAGE, we used a GluA2 variant with three cysteines deleted (see Materials and Methods) (Lau et al., 2013). Amber mutant subunits rescued by BzF could be detected after FLAG-tag purification from whole-cell lysates as a characteristic $\sim 120 \mathrm{kDa}$ band (Fig. 5A). The BzF synthetase also contained a FLAG-tag (Ye et al., 2008), allowing it to act as a transfection and loading control. Upon UV exposure (2-30 min), a band corresponding to a dimer of subunits $(\sim 240 \mathrm{kDa})$ appeared in a time-dependent manner, 
with longer UV exposures resulting in a higher dimer fraction (DF). The formation of dimer in the absence of UV was limited $\left(D F_{\mathrm{S} 729 \mathrm{BzF}}\right.$ pre uv $=1.6 \pm 0.5 \%$; $D F_{\mathrm{S729BzF}} \stackrel{\mathrm{S} 729 \mathrm{BzF}}{=} 15 \stackrel{\text { pre } \mathrm{UV}}{ \pm} 3 \%, n=9 ; p=$ 0.002 ) (Fig. $5 A, B)$. Lysates from cells expressing WT receptors on the background of the tRNA and tRNA synthetase and coincubated with BzF did not yield an appreciable dimer band after $30 \mathrm{~min}$ of $\mathrm{UV}$ exposure $\left(D F_{\mathrm{WT}}\right.$ pre $\mathrm{UV}=3 \pm 1 \%$, $D F_{\mathrm{WT} \text { post } \mathrm{UV}}=5.4 \pm 1.8 \%, n=11, p=$ $0.166)$. Lysates of cells transfected in the same way that were not preincubated in BzF-containing medium expressed a very weak band at $120 \mathrm{kDa}$, which had an average intensity of $4.5 \pm 3 \%(n=4)$ compared with the monomeric BzFcontaining subunit (Fig. $5 C$ ) showing that rescue by $\mathrm{BzF}$ is $\sim 20$-fold more efficient than readthrough, at the level of individual subunits. In these lanes, no band corresponding to dimer molecular weight appeared after $30 \mathrm{~min}$ UV exposure. To check that the crosslinking observed in 3-cysteine-deleted Amber mutants corresponds to receptors that can be photoinactivated, we performed electrophysiological recordings. GluA2 S729TAG $3 \times$ Cys (-) receptors, rescued by coexpression of tRNA, tRNA synthetase, and coincubation with BzF, displayed fast activation and desensitization $\left(k_{\mathrm{des}}=\right.$ $300 \pm 40 \mathrm{~s}^{-1}, n=9$ ) indistinguishable from WT GluA2 on the $3 \times$ Cys $(-)$ background $\left(k_{\mathrm{des}}=230 \pm 13 \mathrm{~s}^{-1}, n=9\right.$; data not shown). Consistent with the GluA2 S729BzF mutant without any cysteine deletions, the $3 \times$ Cys $(-)$ variant could be trapped with a monoexponential time course. In contrast, WT $3 \times$ Cys $(-)$ was essentially insensitive to UV in electrophysiological recordings (Fig. $5 D, E$ ). The rates of the monoexponential inhibition of S729BzF $3 \times$ Cys $(-)$ upon serial exposures to UV illumination were on average similar to receptors containing the native cysteines (time constant for $\mathrm{cu}-$ mulative exposure: $5 \pm 0.3 \mathrm{~s}, n=4$; Fig. $5 D$ ). This biochemical analysis confirms that UV illumination can drive dimerization of GluA2 subunits equipped with BzF in the lower lobes of the LBDs.

\section{Photoinactivation at the G725 and S729 sites is state- independent}

Previous experiments on cysteine mutants at the S729 and G725 sites indicated preferential trapping in the desensitized state (Plested and Mayer, 2009). The faster time resolution of UV exposures allowed us to access different states of the receptor simply by opening the shutter at different points during the record. We exposed patches to UV either before the glutamate pulse (resting state) or during glutamate application (desensitized or active state, depending on the presence or absence of CTZ) and measured the rate of peak current reduction. When comparing active and desensitized states (Fig. 6), the difference in the time constants of current reduction was minor for the S729BzF mutant
B

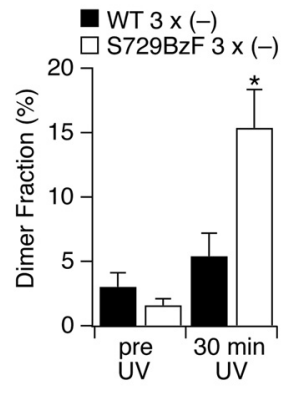

E $10 \mathrm{mM}$ Glu S729BzF
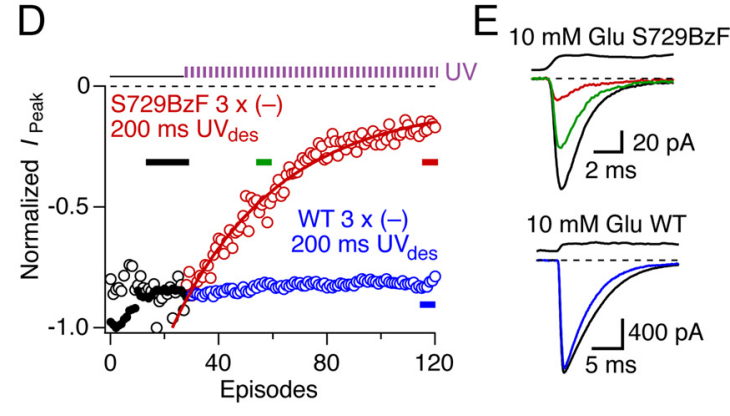

Figure 5. In vivo crosslinking produces UV-dependent dimerization of receptor subunits. $A$, Western blot of GluA2 WT and $\mathrm{S} 729 \mathrm{BzF} 3 \times$ Cys $(-)$ receptors. The experiment was performed in reducing conditions. Monomeric GluA2 bands (M) were detected at $\sim 120 \mathrm{kDa}$. The cells were exposed to UV for time intervals between 2 and 30 min, resulting in an increasing dimeriza-

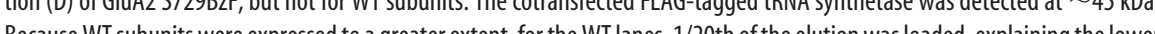

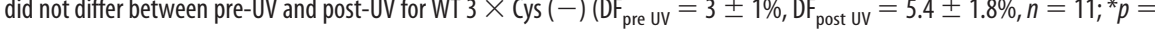
FLAG-tag in the absence of BzF for S729TAG mutants. The band corresponding to the coexpressed FLAG-tagged tRNA synthetase was, however, readily detected, indicating similar loading across S729 lanes. D, On the $3 \times$ Cys (-) background, S729BzF Example traces for GluA2 WT and S729BzF on the $3 \times$ Cys (-) background from the averages of 20 (black) or five traces (green, red, and blue) from the stretches indicated with color-coded bars in the kymogram in $\boldsymbol{D}$.

$(\tau=5.6 \pm 0.2 \mathrm{~s}$ for active state, $n=5-9 ; \tau=3.2 \pm 0.1 \mathrm{~s}$ for desensitized, $n=3-10 ; p=0.35$; Fig. $6 G$ ). The difference in the mean time constants for G725BzF was even smaller but was statistically significant (active: $\tau=4.2 \pm 0.3 \mathrm{~s}$ for $n=3-6$, and desensitized: $\tau=3 \pm 0.1 \mathrm{~s}, n=4-8$ for G725BzF; $p=0.04$, Fig. $6 H)$. These small differences in mean trapping time constants were similar to the differences we saw between individual recordings. Receptor inactivation remained striking, and with similar time constants, when patches were exposed to UV in the resting state, independent of the presence or absence of CTZ for G725BzF $(\tau=3 \pm 2.5 \mathrm{~s}$ without CTZ, $n=7$, and $\tau=1.6 \pm 0.1 \mathrm{~s}$ with CTZ, $n=8)$ and S729BzF $(\tau=1.5 \pm 0.1 \mathrm{~s}, n=3$ without CTZ, and $\tau=2.0 \pm 0.1 \mathrm{~s}$ with CTZ, $n=3)$.

These results suggest that the distinct stereochemistry of BzF allows it to inhibit GluA2 receptors independent of their functional state, in contrast to cysteines placed at the same LBD sites.

\section{State dependence of crosslinking at the sites M721 and Y768}

We hypothesized that the different chemistry of disulfide bonds and $\mathrm{BzF}$ crosslinking underlies the lack of state dependence for the S729BzF and G725BzF mutants. Particularly, the greater length of the BzF side chain, compared with a cysteine, and its multiplicity of possible targets, suggested that state dependence with $\mathrm{BzF}$ is more 
A WT

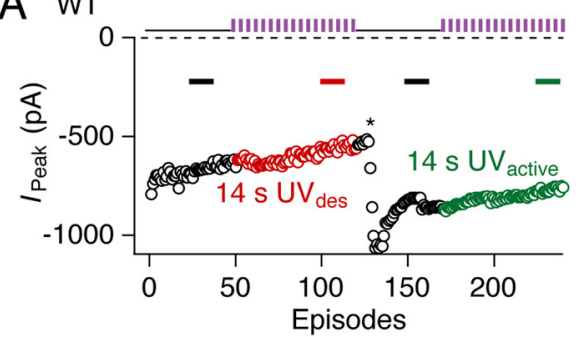

C S729BzF

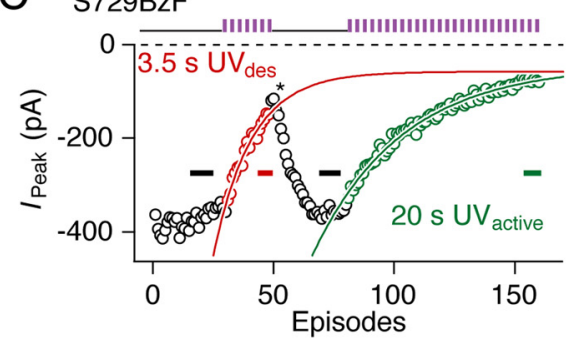

E $\quad$ G725BzF

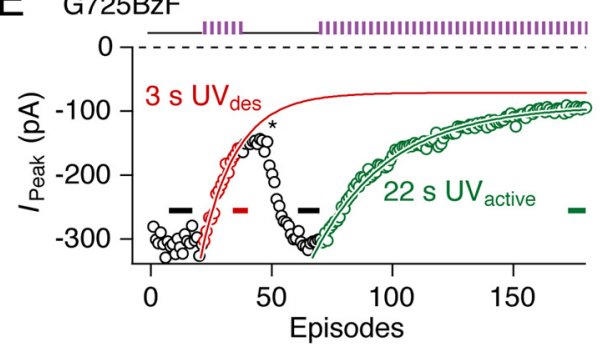

$\mathrm{F}$

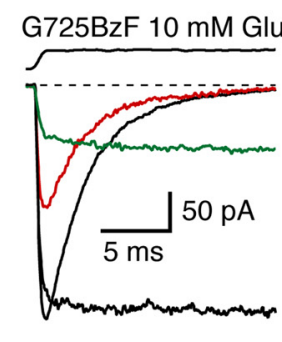

G

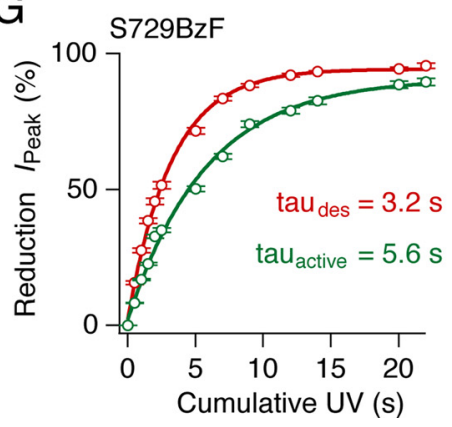

$\mathrm{H}$

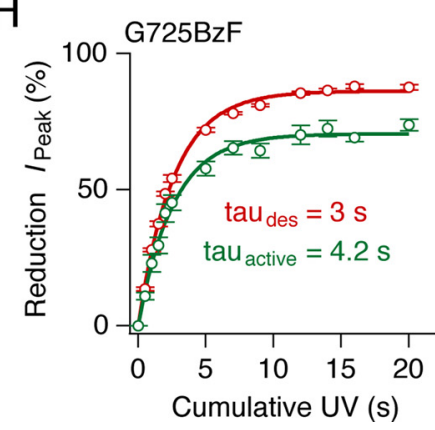

Figure 6. Limited state dependence for S729 and G725. A, Peak current kymogram for WT GluA2 expressed on the tRNA/BzF tRNA synthetase background. Cumulative UV exposures of up to $14 \mathrm{~s}$ ( $200 \mathrm{~ms}$ per episode, shown schematically as a pulse train; violet) did not cause an UV-dependent current reduction in either the desensitized or active state. The active state was isolated by including $100 \mu \mathrm{M}$ CTZ. The asterisk indicates the beginning of the CTZ application. Bars represent groups of traces averaged for $\boldsymbol{B}$. $B$, Average responses to $10 \mathrm{~mm}$ glutamate for WT GluA2, averaged over $\sim 20$ traces from stretches indicated by color-coded bars in A. C, Peak current reduction kymogram for the $5729 \mathrm{Bz}$ mutant. White outlined fits to the current reduction in response to $3.5 \mathrm{~s}$ cumulative UV exposure in the desensitized state (red; $\tau=18$ episodes) and $20 \mathrm{~s}$ exposure in the active state (green; $\tau=42$ episodes) were monoexponential. Symbols and UV exposure schematic are as in $\boldsymbol{A}$. D, As in $\boldsymbol{B}$ for the $\mathrm{S729Bz}$ mutant. Averages of 5-20 traces were taken. $\boldsymbol{E}$, Kymogram, as $\boldsymbol{C}$, for the $\mathrm{G} 725 \mathrm{Bz} F$ mutant. The exponential half-times of peak current reduction were 14 episodes for the desensitized state (red) and 37 episodes for the active state (green). Symbols and UV exposure schematic are as in A. $\boldsymbol{F}$, Averages over groups of traces from stretches indicated by bars in $\boldsymbol{E}$. $\boldsymbol{G}$, Pooled, normalized current reduction curves were fit

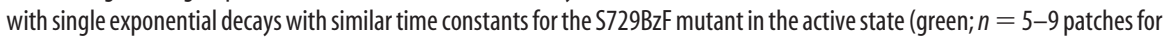
each point) and the desensitized state (red; $3-10$ patches for each point). $\boldsymbol{H}$, As for $\mathbf{G}$, for the $G 725 B z F$ exponential fits to pooled peak current reduction curves for the desensitized state (red; $n=4-8$ patches for each point) and the active state (green; $n=$ 3-6 patches for each point).

likely at sites that are separated by greater extents. Following this logic, we incorporated $\mathrm{BzF}$ at position 721 . When modeled in place of M721 in the active dimer (Armstrong and Gouaux, 2000), the BzF side chain cannot span the separation of the lower lobes (Fig. 7A). In the only available model of the LBD dimer in the desensitized state (Armstrong et al., 2006), the lower lobes of the LBD approach, but the oxygen radical remains $13 \AA$ from the nearest backbone in the opposed subunit (Fig. 7B). In the fulllength, antagonist-bound structure, although this distance is reduced to $8 \AA$ (data not shown), the ketone remains far too distant for crosslinking. Thus, crosslinking with M721BzF would imply either intrasubunit crosslinking or a substantial conformational change upon desensitization (Schauder et al., 2013). We also inserted BzF in place of $Y 768$, a site important for receptor gating at the end of Helix $\mathrm{K}$ (Carbone and Plested, 2012). In the full-length, antagonist-bound crystal structure of GluA2, the $768 \mathrm{BzF}$ side-chain in subunit $\mathrm{A}$ can be oriented to allow its ketone to approach within $6 \AA$ of the electron-rich $C \beta$ of Val666 in subunit D. Crosslinking at this site is likely to result in inhibition (Lau et al., 2013). However, the bond coplanarity required for $\mathrm{H}$-atom abstraction may be difficult to achieve, and the distance is long compared with the preferred $3.1 \AA$ range (Dormán and Prestwich, 1994). BzF is even further from a neighboring subunit in the active dimer (Fig. 7C). However, modeling the BzF side chain in place of $\mathrm{Y} 768$ in the candidate inactive dimer suggests that an intersubunit crosslink might be possible in this state (Fig. 7D). The GluA2 M721BzF and $\mathrm{Y} 768 \mathrm{BzF}$ mutants had similar rates of desensitization to WT channels $\left(k_{\text {des } M 721 \mathrm{BzF}}=\right.$ $101 \pm 6 \mathrm{~s}^{-1}, n=16, p=0.99 ; k_{\mathrm{des}} \mathrm{Y768Bz \textrm {F }}=$ $183 \pm 8 \mathrm{~s}^{-1}, n=19, p=0.31$; Fig. $\left.7 E, F\right)$, but $\mathrm{Y} 768 \mathrm{Bz}$ F had a slower deactivation decay $\left(k_{\text {deact } \mathrm{M} 721 \mathrm{BzF}}=1400 \pm 160 \mathrm{~s}^{-1}, n=7\right.$, $p=0.89 ; k_{\text {deact } \mathrm{Y} 768 \mathrm{BzF}}=700 \pm 60 \mathrm{~s}^{-1}, n=$ $11, p=0.0008$; Fig. $7 G, H$ ). The recovery from desensitization of the M721BzF was noticeably faster than WT $\left(k_{\text {rec }} \mathrm{M} 721 \mathrm{BzF}=\right.$ $67 \pm 9 \mathrm{~s}^{-1}, n=8, p=0.0004$; Fig. $\left.7 I, J\right)$, whereas $\mathrm{Y} 768 \mathrm{BzF}$ did not significantly differ from WT $\left(k_{\text {rec Y768BzF }}=28 \pm 3 \mathrm{~s}^{-1}, n=4\right.$, $p=0.3$ ), consistent with the kinetic changes caused by related mutations in this region (Carbone and Plested, 2012).

In contrast to BzF substitutions at $\mathrm{S} 729$ and G725, the M721BzF mutation exhibited state-dependent reduction of peak current by UV. Consistent with a conformational change that promotes crosslinking upon desensitization, current reduction in the active state $(\tau=140 \pm 40$ episodes, $200 \mathrm{~ms}$ UV per episode, $n=5$ ) was on average $\sim 3.5$-fold slower than that in the desensitized state $(\tau=40 \pm 8$ episodes, $200 \mathrm{~ms}$ UV per episode, $n=7, p=$ 0.04; Fig. $8 A, B)$. State-dependent current reduction was even more pronounced for the Y768BzF mutant than for the M721BzF mutant showing an inverted state dependence (Fig. $8 C-E$ ). This result indicates that current reduction is highly sensitive to the stereochemical environment of BzF in the lower (D2) lobes of the LBD. Peak current was preferentially reduced when UV exposures were made with receptors in the open, fully active state $(\tau=$ 
$49 \pm 6$ episodes, $n=14$ patches, $200-300$ ms UV per episode; Fig. $8 C, E$ ) or in the resting state stabilized by CTZ $(\tau=26 \pm 5$ episodes, $n=4$; Fig. $8 D, E)$. Surprisingly, given our expectation from the candidate inactivated dimer of intersubunit crosslinking between lower lobes, the $768 \mathrm{BzF}$ mutant was almost unaffected by UV in desensitized states, being trapped at least an order of magnitude more slowly $(\tau=$ $400 \pm 90$ episodes, $n=8 ; p=0.006$ vs active state, $p=0.005$ vs resting state). The time constant of trapping (and thus the degree of state dependence) is probably underestimated in this case because of our inability to hold patches stable for the requisite amount of time $(>10 \mathrm{~min})$ to accurately measure such slow modification. State dependence did not depend on the order in which the states were assessed, with strong current reduction by UV either if exposures were first made in the desensitized state or if this step was omitted. To further investigate whether the reduction in current observed was the result of intersubunit covalent bonds, we performed biochemical experiments on the Y768BzF mutant but did not detect UV-driven dimerization even after $30 \mathrm{~min}$ exposure (dimer fraction before UV $=$ $3.5 \pm 1.2 \%$, dimer fraction after UV $=$ $3.3 \pm 0.7 \% n=3$ blots; Fig. $8 F$ ), suggesting that any crosslinking between subunits was limited. In this experiment, faint dimer bands were seen for WT $3 \times$ Cys $(-)$ channels, serving to illustrate the extent of variability while using the same conditions. Critically, WT receptors never showed an UV-driven increase in dimer band intensity (Fig. 5).

\section{Discussion}

Effective rescue of Amber mutant receptors by photoactivatable UAAs GluA2 receptors carrying Amber mutations in D2 (Fig. 1) were rescued by photoactivatable UAAs (AzF or BzF). Desensitization was dramatically faster when AzF was incorporated in place of S729, arguing for specific incorporation of UAAs because S729BzF kinetics were only slightly faster than those of WT. Upon UV illumination, BzF forms a diradical, which reacts with $\mathrm{C}-\mathrm{H}$ bonds to form a covalent adduct. If the crosslinking reaction fails because of the absence of nearby $\mathrm{C}-\mathrm{H}$ bonds, BzF returns to the ground state and can be excited again (Dormán and Prestwich, 1994; Tanaka et al., 2008). In contrast, AzF activation is irreversible, with a range of targets, including nucleophiles (Knowles, 1972) and is also triggered by ambient daylight (Grunbeck et al., 2011). Furthermore, nonspecific crosslinking and photodamage are more likely when using AzF because it is excited at shorter wavelengths $(<310 \mathrm{~nm}$; Chin et al., 2002b; Tanaka et al., 2008) thanBzF (350-365 nm; Dormán and Prestwich, 1994).
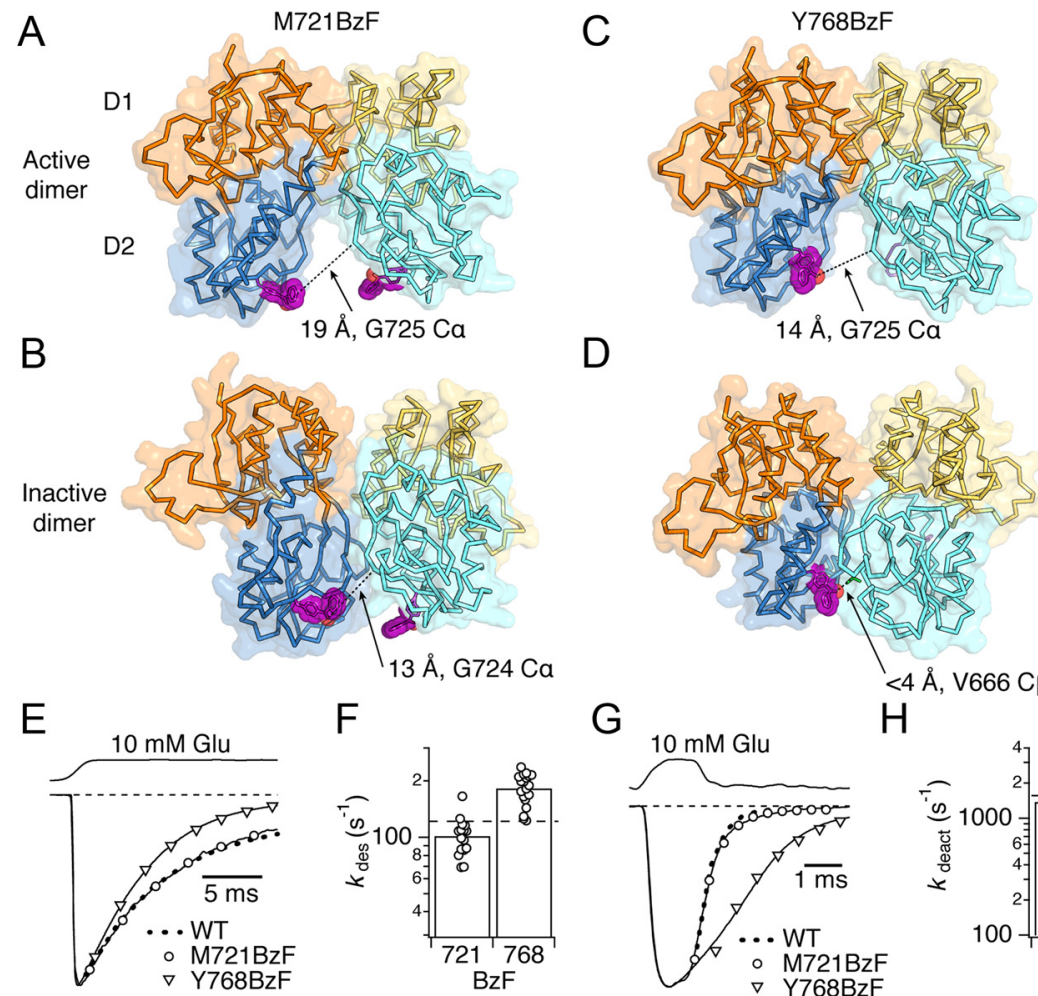

$\mathrm{H}$

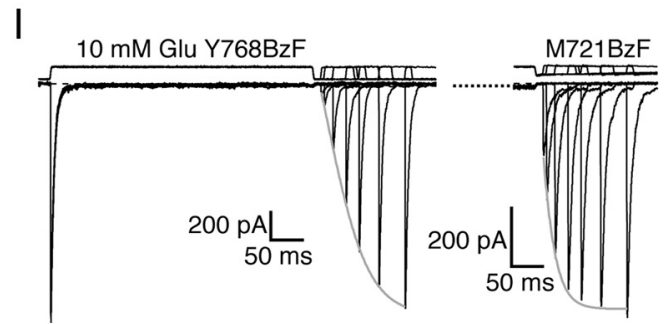

$\mathrm{J}$

Figure 7. Properties of the M721BzF and $\mathrm{Y768BzF}$ mutants. $A$, The M721BzF mutation modeled into the active $L B D$ dimer of GluA2 (PDB ID: 1FTJ, chains A and C). Same color scheme as in Figure 1, with BzF indicated in violet. Dashed line indicates the distance to the nearest $C-H$ bond in the opposite subunit. $B$, The $M 721 B z F$ mutation modeled into the candidate desensitized dimer (PDB ID: 2l3W). C, The Y768BzF mutation modeled into active LBD dimer of GluA2. D, The Y768BzF mutation modeled into the candidate desensitized dimer. $\boldsymbol{E}$, Normalized responses to $400 \mathrm{~ms}$ pulses of $10 \mathrm{~mm}$ glutamate for M721BzF and Y768BzF mutants. The desensitization rates in the examples shown were $k_{\text {des }}=134 \mathrm{~s}^{-1}$ for M721BzF (circles) and $k_{\text {des }}=185 \mathrm{~s}^{-1}$ for Y768BzF (triangles). An equivalent decay from a WT patch is drawn as a dashed line. $\boldsymbol{F}$, Summary of desensitization rates of receptors carrying BzF at positions M721 ( $\left.k_{\text {des }}=101 \pm 6 s^{-1}, n=16 ; p=0.99\right)$ and $Y 768\left(k_{\text {des }}=183 \pm 6 s^{-1}, n=19 ; p=0.31\right)$. Dashed line indicates the wild-type rate. $G$, Normalized responses to nominal 1 ms pulses of $10 \mathrm{~mm}$ glutamate for the $M 721 \mathrm{BzF}$ and Y768BzF mutants. The deactivation rates for the examples plotted here were $k_{\text {deact }}=1961 \mathrm{~s}^{-1}$ for M721BzF (circles) and $k_{\text {deact }}=$ $617 \mathrm{~s}^{-1}$ for $\mathrm{Y768BzF}$ (triangles). A WT trace is shown as a dotted line for comparison. $\boldsymbol{H}$, Summary of deactivation rates of M721BzF mutant $\left(k_{\text {deact }}=1400 \pm 160 \mathrm{~s}^{-1}, n=7 ; p=0.89\right)$ and the Y768BzF mutant $\left(k_{\text {des }}=700 \pm 60 \mathrm{~s}^{-1}, n=11: p=0.0008\right)$. Dashed line indicates the wild-type rate. $I$, Example time courses of recovery from desensitization for M721BzF and $Y 768 \mathrm{BzF}$ mutants (7 overlaid traces for each panel). J, Pooled recovery data for M721BzF $\left(k_{\text {rec }}=67 \pm 9 s^{-1}, n=8: p=0.0004\right)$ and Y768BzF ( $\left.k_{\text {rec }}=28 \pm 3 s^{-1}, n=4 ; p=0.3\right)$. WT GluA2 recovery is shown as a dotted line.

\section{Irreversible inactivation of GluA2 Amber mutants by UV light}

GluA2 receptors harboring BzF in D2 were irreversibly photoinactivated upon UV irradiation, resulting in a loss of peak current. All BzF mutants were UV-sensitive, although substitutions at M721 and Y768 were only inhibited in particular functional states. We were able to control the trapping rate by varying or stopping the UV illumination, eliminating rundown as a cause of the current reduction. The peak current reduction was invariably monoexponential, suggesting that a single crosslinking event is enough to substantially inactivate the receptor. We assume that either subsequent crosslinking in the complex is silent or the tetramer is driven into a nonsymmetric arrangement whose ge- 
A A2 M721BzF

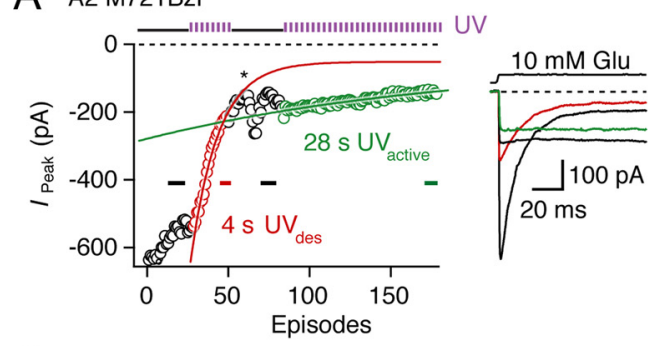

C A2 Y768BzF

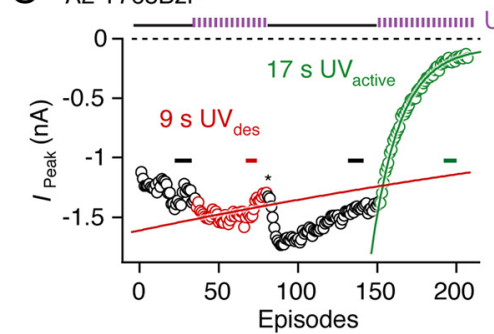

D

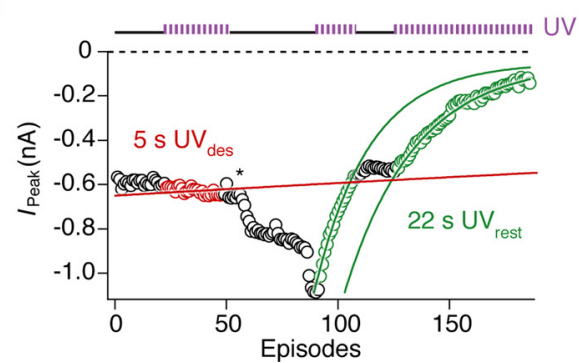

B

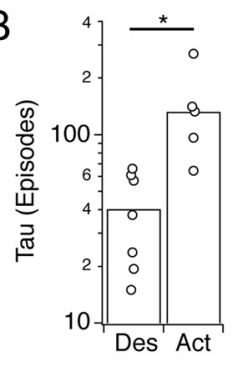

$\mathrm{E}$

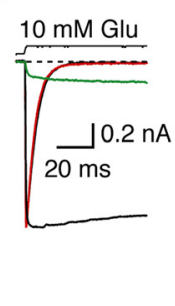

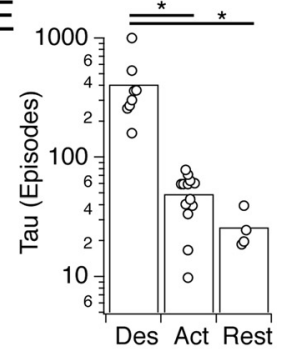

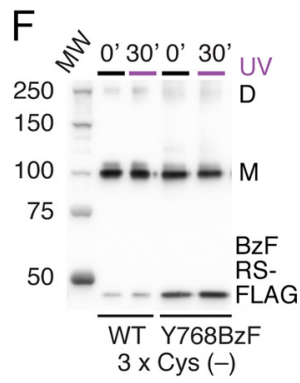

Figure 8. M721BzF and $\mathrm{Y768BzF}$ mutants are state-dependent. $\boldsymbol{A}$, Peak current reduction kymogram for M721BzF for the desensitized state (red; 200 ms UV exposure per episode) and the active state (green; 300 ms UV exposures each episode). Monoexponential fits to the current reduction (fixed to a common minimum; for this example, $\tau_{\text {des }}=20$ episodes; $\tau_{\text {active }}=270$ ). Cumulative exposure of UV ( 200 or $300 \mathrm{~ms}$ per episode; shown schematically as a pulse train, violet) was $4 \mathrm{sin}$ the desensitized state

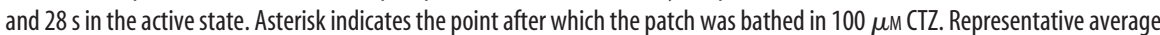
currents activated by $10 \mathrm{~mm}$ glutamate are shown, color-coded according to the bars in the kymogram. $\boldsymbol{B}$, Summary of time constants for peak current reduction (in episodes) for the M721BzF mutant with UV exposures of $200 \mathrm{~ms}$ for active and desensitized states $\left(\tau_{\text {des }}=40 \pm 8, n=7 ; \tau_{\text {active }}=140 \pm 40, n=5 ;{ }^{*} p=0.04\right.$ ). C, Peak current reduction kymogram for $\mathrm{Y} 768 \mathrm{BzF}$ for the desensitized state (red; 200 ms UV exposure per episode) and the active state (green; 300 ms UV exposures each episode). Monoexponential fits to the current reduction (fixed to a common minimum; $\tau_{\text {des }}=535$ episodes; $\tau_{\text {active }}=17$ for this example). Symbols and UV exposure schematic are as in $\boldsymbol{A}$. Cumulative UV exposure was $9 \mathrm{~s}$ in the desensitized state and $17 \mathrm{~s}$ in the active state. Representative average currents activated by $10 \mathrm{~mm}$ glutamate are shown, color-coded according to the bars in the kymogram. D, Peak current reduction kymogram for Y768BzF with 200 ms UV exposures each episode in the desensitized (red) and resting states (green; in the presence of $(T Z$ ). Monoexponential fits to the current reduction (fixed to a common minimum; for this example, $\tau_{\text {des }}=1000$ episodes; $\tau_{\text {active }}=25$ ). Symbols and UV exposure schematic are as in $\boldsymbol{A}$. Cumulative UV exposure was $5 \mathrm{sin}$ the desensitized state and $22 \mathrm{~s}$ in the active state. There is a brief interval in the resting state trapping when no UV exposures were made (black circles). $\boldsymbol{E}$, Summary of time constants for peak current reduction for the Y768BzF mutant for desensitized $(\tau=400 \pm$ 90 episodes, $n=8 ; p=0.006$ vs active state: ${ }^{*} p=0.005$ vs resting state) and resting states ( $\tau=26 \pm 5$ episodes, $n=4$; both $200 \mathrm{~ms}$ exposures per episode). For the active state, we saw no difference in the trapping time constant for 200 or $300 \mathrm{~ms}$ UV exposures per episode, and these were pooled ( $\tau=49 \pm 6$ episodes, $n=14$ patches). $\boldsymbol{F}$, Western blot (anti-FLAG antibody) for WT and Y768BzF receptors before and after a 30 min UV exposure. M, Monomer; D, dimer; MW, molecular weight marker.

G725BzF homomeric mutants after trapping. Assuming binomial assembly, a high probability of random amino acid insertion (e.g., 45\%) is required to produce a $5 \%$ subpopulation of receptors completely lacking BzF side chains. We observed a much lower "readthrough" in biochemical experiments ( $\sim 5 \%$, see below), suggesting that the residual current is an intrinsic property of the trapped receptors.

\section{UV-induced photocrosslinking of subunits in live cells}

Full-length rescued subunits $(\sim 120 \mathrm{kDa})$ were only detected in cells that were incubated in a BzF-containing medium, but not in controls lacking BzF. Thus, at least at position S729, background readthrough is inefficient, with incorporation of BzF by the exogenous synthetase being 10 - to 20 -fold more efficient than that of endogenous amino acids. Irradiating live cells with UV generated dimer bands $(\sim 240 \mathrm{kDa})$ in a time-dependent fashion, providing strong evidence that BzF groups can crosslink subunits covalently, as previously described for cytoplasmic proteins (Farrell et al., 2005). There are several possible reasons for the low efficiency of crosslinking. The inhibition seen in functional experiments could be in part due to crosslinking onto neighboring subunits and in part due to intrasubunit recombination of BzF, which would tend to deplete the dimer fraction. We cannot exclude that immature intracellular forms of the receptor, such as dimers, could be crosslinked by UV, although for the overexpression intervals we used $(72 \mathrm{~h})$, dimers should represent at most a minor fraction of the GluA2 subunits in an entire cell (Shanks et al., 2010). Furthermore, crosslinking is site specific because Y768BzF largely failed to dimerize. We estimate that the UV illumination in biochemical experiments was $\sim 100$-fold less intense than in electrophysiological recordings. This discrepancy almost certainly meant that crosslinking was incomplete. Trapping intervals longer than 30 min produced nonspecific dimers in WT controls, possibly due to hypoxia or photo ometry prevents further crosslinking. It is equally possible that multiple UV-driven crosslinking events occur independently (perhaps within subunits), combining to produce the monoexponential loss of current. If multiple forms of crosslinking occur, they should do so with the same rate; otherwise, the decay would not be apparently monoexponential. A single intersubunit crosslink that inactivates both subunits seems reasonable on the basis of previous crosslinking at this site and our biochemical results indicating dimerization with S729BzF. We note that receptors with two subunits active have a low open probability and $\sim 10 \%$ of the conductance of fully active channels (Rosenmund et al., 1998), consistent with the $\sim 5 \%$ residual activity of $S 729 \mathrm{BzF}$ or damage. A single BzF crosslinking event in a mature tetramer might drive a nonsymmetric arrangement of the LBD layer, blocking further crosslinking between subunits, which would further contribute to the small dimer fraction (15\%). This situation is analogous to that in some cysteine mutants, for which only two of the four subunits in a complex can crosslink, and which show incomplete crosslinking ( $\sim 30 \%$; Lau et al., 2013).

\section{Investigating receptor structure and dynamics with photoinactivation}

The state dependence we observed in the Y768BzF and M721BzF mutants allows us to map putative crosslinking events onto struc- 
tural templates of functional states, with two caveats: (1) the exact geometry of BzF trapping is unknown, given potential crosslinking sites in any of the four subunits in the complex; and (2) models of the tetramer of LBDs in either the active or desensitized states are lacking.

Photoinactivation of M721BzF was most efficient in the desensitized state, which is inconsistent with the putative desensitized dimer crystal structure (Armstrong et al., 2006). In this structure, the ketone radical cannot approach closer than $13 \AA$ from the nearest backbone in the opposing subunit (Fig. 7B). We were not able to express the M721BzF mutant well enough to detect multimeric bands in biochemical studies. Developing alternative genetic targeting methods (including packaging receptor, synthetase, and tRNA coding sequences into a single vector) may help to increase yields of rescued receptors to address this problem. Consistent with a preference for intrasubunit recombination and the idea that receptors incorporating naive subunits are better inactivated by intersubunit crosslinks, GluA2 M721BzF was relatively poor at inactivating heteromeric receptors (mean reduction in current: $30 \pm 4 \%, n=4$ patches; data not shown).

In the inactive dimer structure (Armstrong et al., 2006), the $\mathrm{BzF}$ side chain can be arranged so that the oxygen radical comes within $4 \AA$ of the $\mathrm{C} \beta$ of Val666, with good likelihood of the necessary coplanarity (Dormán and Prestwich, 1994) (Fig. 7D), but notably, functional crosslinking was negligible during receptor desensitization, suggesting that such an arrangement is not often obtained in the desensitized state. Crosslinking within subunits seems likely for $\mathrm{Y} 768 \mathrm{BzF}$, given that this mutant was potently and selectively photoinactivated in the active state, although the BzF side chain is poorly placed to reach neighboring subunits in this conformation (Fig. $7 C$ ), or the antagonist-bound crystal structure of the full-length receptor. The $\mathrm{Y} 768 \mathrm{BzF}$ mutant expressed readily but did not give dimer bands in biochemical experiments. Therefore, state dependence likely arises from deformation of the lower lobe of the LBD upon desensitization, reducing intrasubunit crosslinking for the $768 \mathrm{BzF}$ mutant while increasing opportunities for M721BzF to recombine. Such a deformation, perhaps resembling movements of the lower lobe deduced from molecular dynamics simulations (Yao et al., 2013), might also explain the common regulation of gating and desensitization by spatially distinct point mutations in the lower lobe of the LBD (Carbone and Plested, 2012). Changes in D2 conformation are also expected if AMPA receptor LBDs must accommodate twisting (relative to the transmembrane domain) on the scale of that seen in kainate receptors (Schauder et al., 2013). Further screening of sites within lower lobe of iGluRs should help to resolve this picture.

\section{Implications for inactivating native receptors}

Several methods have been used to inactivate glutamate receptors in vivo, including RNAi and ANQX (Adesnik et al., 2005; Tracy et al., 2011). Despite notable advantages (e.g., targeting exclusively surface receptors, in the case of ANQX), these methods either lack cellular specificity or develop slowly (over hours or days). Using BzF photoinactivation, the majority of AMPA receptors within a targeted cell could be inactivated within $\sim 10 \mathrm{~s}$, independent of their functional state. Receptors incorporating naive subunits were also inactivated, so overexpression of one BzF-containing subtype could target native heteromultimeric channels on a WT background, complementing "molecular replacement" approaches (Granger et al., 2013). Heteromeric AMPA receptors dominated in our coexpression experiments, indicating that BzF-containing subunits are as well expressed as WT subunits.
Heteromeric receptors containing BzF at positions 725 or 729 in GluA2 showed a smaller peak current reduction than homomers. We cannot exclude the possibility that trapping induces a conformation in which glutamate remains a partial agonist, or that only the subunits containing BzF groups were inactivated, through intrasubunit crosslinking. However, photoinactivation did not change the IV relation (which depends on polyamine block and subunit composition), rendering unlikely the possibility that a subpopulation of receptors with a particular subunit stoichiometry is preferentially trapped. Partially inactivated receptors should activate with subconductance openings, which might be expected to show different block by polyamines. However, such an effect might be too small to detect, and unraveling the responses of a mixture of receptors with distinct conductances, given that unmodified AMPA receptors also activate with subconductances (Rosenmund et al., 1998; Carbone and Plested, 2012), could prove challenging.

\section{References}

Adesnik H, Nicoll RA, England PM (2005) Photoinactivation of native AMPA receptors reveals their real-time trafficking. Neuron 48:977-985. CrossRef Medline

Armstrong N, Gouaux E (2000) Mechanisms for activation and antagonism of an AMPA-sensitive glutamate receptor: crystal structures of the GluR2 ligand binding core. Neuron 28:165-181. CrossRef Medline

Armstrong N, Jasti J, Beich-Frandsen M, Gouaux E (2006) Measurement of conformational changes accompanying desensitization in an ionotropic glutamate receptor. Cell 127:85-97. CrossRef Medline

Beene DL, Dougherty DA, Lester HA (2003) Unnatural amino acid mutagenesis in mapping ion channel function. Curr Opin Neurobiol 13: 264-270. CrossRef Medline

Carbone AL, Plested AJ (2012) Coupled control of desensitization and gating by the ligand binding domain of glutamate receptors. Neuron 74:845857. CrossRef Medline

Chin JW, Martin AB, King DS, Wang L, Schultz PG (2002a) Addition of a photocrosslinking amino acid to the genetic code of Escherichia coli. Proc Natl Acad Sci U S A 99:11020-11024. CrossRef Medline

Chin JW, Santoro SW, Martin AB, King DS, Wang L, Schultz PG (2002b) Addition of $p$-azido-L-phenylalanine to the genetic code of Escherichia coli. J Am Chem Soc 124:9026-9027. CrossRef Medline

Chin JW, Cropp TA, Anderson JC, Mukherji M, Zhang Z, Schultz PG (2003) An expanded eukaryotic genetic code. Science 301:964-967. CrossRef Medline

Cornell R (1989) Chemical cross-linking reveals a dimeric structure for CTP:phosphocholine cytidylyltransferase. J Biol Chem 264:9077-9082. Medline

Das U, Kumar J, Mayer ML, Plested AJ (2010) Domain organization and function in GluK2 subtype kainate receptors. Proc Natl Acad Sci U S A 107:8463-8468. CrossRef Medline

Ding S, Horn R (2001) Slow photo-cross-linking kinetics of benzophenonelabeled voltage sensors of ion channels. Biochemistry 40:10707-10716. CrossRef Medline

Dormán G, Prestwich GD (1994) Benzophenone photophores in biochemistry. Biochemistry 33:5661-5673. CrossRef Medline

Farrell IS, Toroney R, Hazen JL, Mehl RA, Chin JW (2005) Photo-crosslinking interacting proteins with a genetically encoded benzophenone. Nat Methods 2:377-384. CrossRef Medline

Gielen M, Le Goff A, Stroebel D, Johnson JW, Neyton J, Paoletti P (2008) Structural rearrangements of NR1/NR2A NMDA receptors during allosteric inhibition. Neuron 57:80-93. CrossRef Medline

Granger AJ, Shi Y, Lu W, Cerpas M, Nicoll RA (2013) LTP requires a reserve pool of glutamate receptors independent of subunit type. Nature 493: 495-500. CrossRef Medline

Grunbeck A, Huber T, Sachdev P, Sakmar TP (2011) Mapping the ligandbinding site on a G-protein-coupled receptor (GPCR) using genetically encoded photocrosslinkers. Biochemistry 50:3411-3413. CrossRef Medline

Hino N, Hayashi A, Sakamoto K, Yokoyama S (2006) Site-specific incorporation of non-natural amino acids into proteins in mammalian cells with an expanded genetic code. Nat Protoc 1:2957-2962. CrossRef Medline 
Horn R, Ding S, Gruber HJ (2000) Immobilizing the moving parts of voltagegated ion channels. J Gen Physiol 116:461-476. CrossRef Medline

Knowles JR (1972) Photogenerated reagents for biological receptor-site labeling. Acc Chem Res 5:155-160. CrossRef

Lau AY, Salazar H, Blachowicz L, Ghisi V, Plested AJ, Roux B (2013) A conformational intermediate in glutamate receptor activation. Neuron 79:492-503. CrossRef Medline

Liu W, Alfonta L, Mack AV, Schultz PG (2007a) Structural basis for the recognition of para-benzoyl-L-phenylalanine by evolved aminoacyltRNA synthetases. Angew Chem Int Ed Engl 46:6073-6075. CrossRef Medline

Liu W, Brock A, Chen S, Chen S, Schultz PG (2007b) Genetic incorporation of unnatural amino acids into proteins in mammalian cells. Nat Methods 4:239-244. CrossRef Medline

Naganathan S, Ye S, Sakmar TP, Huber T (2013) Site-specific epitope tagging of $\mathrm{G}$ protein-coupled receptors by bioorthogonal modification of a genetically encoded unnatural amino acid. Biochemistry 52:1028-1036. CrossRef Medline

Pless SA, Ahern CA (2013) Unnatural amino acids as probes of ligandreceptor interactions and their conformational consequences. Annu Rev Pharmacol Toxicol 53:211-229. CrossRef Medline

Plested AJ, Mayer ML (2009) AMPA receptor ligand binding domain mobility revealed by functional cross linking. J Neurosci 29:11912-11923. CrossRef Medline

Robert A, Armstrong N, Gouaux JE, Howe JR (2005) AMPA receptor binding cleft mutations that alter affinity, efficacy, and recovery from desensitization. J Neurosci 25:3752-3762. CrossRef Medline

Rosenmund C, Stern-Bach Y, Stevens CF (1998) The tetrameric structure of a glutamate receptor channel. Science 280:1596-1599. CrossRef Medline

Schauder DM, Kuybeda O, Zhang J, Klymko K, Bartesaghi A, Borgnia MJ, Mayer ML, Subramaniam S (2013) Glutamate receptor desensitization is mediated by changes in quaternary structure of the ligand binding domain. Proc Natl Acad Sci U S A 110:5921-5926. CrossRef Medline

Shanks NF, Maruo T, Farina AN, Ellisman MH, Nakagawa T (2010) Contribution of the global subunit structure and stargazin on the maturation of AMPA receptors. J Neurosci 30:2728-2740. CrossRef Medline

Sobolevsky AI, Rosconi MP, Gouaux E (2009) X-ray structure, symmetry and mechanism of an AMPA-subtype glutamate receptor. Nature 462: 745-756. CrossRef Medline

Tanaka Y, Bond MR, Kohler JJ (2008) Photocrosslinkers illuminate interactions in living cells. Mol Biosyst 4:473-480. CrossRef Medline

Tracy TE, Yan JJ, Chen L (2011) Acute knockdown of AMPA receptors reveals a trans-synaptic signal for presynaptic maturation. EMBO J 30: 1577-1592. CrossRef Medline

Traynelis SF, Wollmuth LP, McBain CJ, Menniti FS, Vance KM, Ogden KK, Hansen KB, Yuan H, Myers SJ, Dingledine R (2010) Glutamate receptor ion channels: structure, regulation, and function. Pharmacol Rev 62:405496. CrossRef Medline

Wang L, Brock A, Herberich B, Schultz PG (2001) Expanding the genetic code of Escherichia coli. Science 292:498-500. CrossRef Medline

Wang W, Takimoto JK, Louie GV, Baiga TJ, Noel JP, Lee KF, Slesinger PA, Wang L (2007) Genetically encoding unnatural amino acids for cellular and neuronal studies. Nat Neurosci 10:1063-1072. CrossRef Medline

Weston MC, Gertler C, Mayer ML, Rosenmund C (2006a) Interdomain interactions in AMPA and kainate receptors regulate affinity for glutamate. J Neurosci 26:7650-7658. CrossRef Medline

Weston MC, Schuck P, Ghosal A, Rosenmund C, Mayer ML (2006b) Conformational restriction blocks glutamate receptor desensitization. Nat Struct Mol Biol 13:1120-1127. CrossRef Medline

Yao Y, Belcher J, Berger AJ, Mayer ML, Lau AY (2013) Conformational analysis of NMDA receptor GluN1, GluN2, and GluN3 ligand-binding domains reveals subtype-specific characteristics. Structure 21:17881799. CrossRef Medline

Ye S, Köhrer C, Huber T, Kazmi M, Sachdev P, Yan EC, Bhagat A, RajBhandary UL, Sakmar TP (2008) Site-specific incorporation of keto amino acids into functional $\mathrm{G}$ protein-coupled receptors using unnatural amino acid mutagenesis. J Biol Chem 283:1525-1533. CrossRef Medline

Ye S, Huber T, Vogel R, Sakmar TP (2009) FTIR analysis of GPCR activation using azido probes. Nat Chem Biol 5:397-399. CrossRef Medline

Ye S, Zaitseva E, Caltabiano G, Schertler GF, Sakmar TP, Deupi X, Vogel R (2010) Tracking G-protein-coupled receptor activation using genetically encoded infrared probes. Nature 464:1386-1389. CrossRef Medline

Ye S, Riou M, Carvalho S, Paoletti P (2013) Expanding the genetic code in Xenopus laevis oocytes. Chembiochem 14:230-235. CrossRef Medline 